Draft version MARCh 31, 2018

Preprint typeset using $\mathrm{IAT}_{\mathrm{E}} \mathrm{X}$ style emulateapj v. 5/2/11

\title{
MAGNETOHYDRODYNAMIC-PARTICLE-IN-CELL METHOD FOR COUPLING COSMIC RAYS WITH A THERMAL PLASMA: APPLICATION TO NON-RELATIVISTIC SHOCKS
}

\author{
Xue-Ning Bai ${ }^{1,3}$, Damiano Caprioli ${ }^{2}$, Lorenzo Sironi ${ }^{1,4}$, Anatoly Spitkovsky ${ }^{2}$ \\ ${ }^{1}$ Institute for Theory and Computation, Harvard-Smithsonian Center for Astrophysics, 60 Garden St., MS-51, Cambridge, MA 02138 \\ and \\ ${ }^{2}$ Department of Astrophysical Sciences, Peyton Hall, Princeton University, Princeton, NJ 08544 \\ Draft version March 31, 2018
}

\begin{abstract}
We formulate a magnetohydrodynamic-particle-in-cell (MHD-PIC) method for describing the interaction between collisionless cosmic ray (CR) particles and a thermal plasma. The thermal plasma is treated as a fluid, obeying equations of ideal MHD, while CRs are treated as relativistic Lagrangian particles subject to the Lorentz force. Backreaction from CRs to the gas is included in the form of momentum and energy feedback. In addition, we include the electromagnetic feedback due to CRinduced Hall effect that becomes important when the electron-ion drift velocity of the background plasma induced by CRs approaches the Alfvén velocity. Our method is applicable on scales much larger than the ion inertial length, bypassing the microscopic scales that must be resolved in conventional PIC methods, while retaining the full kinetic nature of the CRs. We have implemented and tested this method in the Athena MHD code, where the overall scheme is second-order accurate and fully conservative. As a first application, we describe a numerical experiment to study particle acceleration in non-relativistic shocks. Using a simplified prescription for ion injection, we reproduce the shock structure and the CR energy spectra obtained with more self-consistent hybrid-PIC simulations, but at substantially reduced computational cost. We also show that the CR-induced Hall effect reduces the growth rate of the Bell instability and affects the gas dynamics in the vicinity of the shock front. As a step forward, we are able to capture the transition of particle acceleration from non-relativistic to relativistic regimes, with momentum spectrum $f(p) \propto p^{-4}$ connecting smoothly through the transition, as expected from the theory of Fermi acceleration.

Subject headings: methods: numerical — magnetohydrodynamics — plasmas — instabilities — acceleration of particles — shock waves
\end{abstract}

\section{INTRODUCTION}

Cosmic rays (CRs) are charged particles that possess much higher energy than particles in the surrounding medium. They are a dynamically important constituent in the Galaxy, where their energy density is in rough equipartition with gas pressure and magnetic pressure. They also provide key observational diagnostics for astrophysical phenomena via non-thermal radiation. The origin and transport of CRs are among the forefront topics of astrophysics, and involve collisionless kinetic effects with complex non-linear interactions with magnetic fields and background thermal plasma.

Particle-in-cell (PIC) methods (e.g., Birdsall \& Langdon 2005), which iteratively move particles and update the electromagnetic fields, have been commonly employed to study the plasma physics of CRs from first principles. Fully kinetic PIC codes treat both electrons and ions as particles (e.g., Fonseca et al. 2002, Bowers et al. 2008, Spitkovsky 2005). While being the most self-consistent method that rigorously solves for everything from plasma oscillations to the propagation of light waves, the main disadvantage is that it has to resolve the microscopic plasma scale: the plasma (electron) skin

\footnotetext{
xbai@cfa.harvard.edu

3 NASA Hubble Fellow

4 NASA Einstein Fellow
}

depth $c / \omega_{p e} \sim 5.4 \times 10^{5} \mathrm{~cm}\left(\mathrm{n} / \mathrm{cm}^{-3}\right)^{-1 / 2}$, where $\omega_{p e}$ is the electron plasma frequency. Moreover, the ion scale is well separated from the electron scale. Even with artificially reduced ion-to-electron mass ratio, properly capturing the physics at both scales still demands tremendous computational cost.

An alternative approach, called the hybrid-PIC, treats all ions as kinetic particles, while electrons are included as a neutralizing, massless and conducting fluid with a prescribed equation of state (e.g., Lipatov 2002; Winske et al. 2003; Gargaté et al. 2007; Kunz et al. 2014a). By compromising the electron-scale physics, the physics at the ion-scale can be well captured with much reduced computational cost, and this method has been successfully applied to study a wide range of laboratory, space physics and astrophysical problems. The hybrid-PIC approach again is limited by the requirement of resolving the characteristic ion scale, the ion inertial length $c / \omega_{p i} \sim 2.3 \times 10^{7} \mathrm{~cm}\left(n / \mathrm{cm}^{-3}\right)^{-1 / 2}$, where $\omega_{p i}$ is the ion plasma frequency and $n$ is the ion number density. This scale is typically much smaller than the CR gyro radius. The latter scale is particularly relevant for resonant scattering of CRs, yet it becomes progressively more difficult to accommodate using the hybrid-PIC method as the CR gyroradius becomes much larger than the ion inertial length with increasing CR energy.

Another approach is to treat CRs as non-thermal test 
particles, embedded in a thermal plasma (gas) described by magnetohydrodynamics (e.g. Lehe et al. 2009; Beresnyak et al. 2011). Being test particles, CRs only passively respond to the electromagnetic field carried by the gas. On the other hand, CRs are well known to influence the dynamics of the background gas and magnetic fields, which in turn affect their propagation (e.g., Kulsrud \& Pearce 1969; Skilling 1975; Parker 1992).

In this paper, we consider another approach, which we call the MHD-PIC method. We treat only CRs as particles using the conventional PIC technique, while the rest of the thermal plasma (ions and electrons) is treated as gaseous fluid described by magnetohydrodynamics (MHD). Different from the test particle approach, the CR feedback to the gas is included following a rigorous formulation that enables the mutual interactions between the thermal gas and CRs. With CRs treated as particles, kinetic effects of CRs can be fully captured. With fluid treatment of the thermal plasma, we avoid resolving microscopic scales, allowing us to accommodate much larger and even macroscopic scales in our simulations with only modest computational cost ${ }^{3}$.

We note that similar methods as we describe in this paper have been proposed and implemented to study the CR streaming instabilities. Zachary (1987) derived the formulation from a plasma point of view (published in Zachary \& Cohen 1986), with CR feedback reflected in the momentum and energy equations as external Lorentz force and heating/cooling due to CR charge and current. Lucek \& Bell (2000) used the same formulation and discovered in their simulations what was later termed the Bell instability (Bell 2004), which is a non-resonant instability driven by the CR current. Reville \& Bell (2012) implemented the same method and studied in detail the filamentation instability of Bell (2005). Reville \& Bell (2013) and Bell et al. (2013) adopted the same MHD formulation, but developed a Vlasov-Fokker-Planck approach for CRs with approximate closure relations (instead of using particles) to study CR diffusion, escape and magnetic field amplification in the upstream of collisionless shocks.

In this work, we re-derive the formulation rigorously in the MHD framework, and further show that there is an additional $\mathrm{CR}$ feedback term which modifies the induction and energy equations. For sufficiently strong CR currents, this term, which we call the CR-induced Hall term, may appreciably affect the growth rate of the Bell instability and become important in the upstream fluid as it approaches the shock front. We have implemented the MHD-PIC formalism described in this paper into the state-of-the-art, fully parallelized MHD code Athena (Stone et al. 2008). This opens up a variety of CR-related problems that can be addressed using non-linear computer simulations, especially the long-term evolution of non-relativistic shocks, to be introduced in Section 1.1.

\footnotetext{
3 While the thermal plasma can be collisionless or weakly collisional, we employ the MHD framework which implicitly assumes efficient inter-particle "collisions" via plasma instabilities at microscopic scales so that the plasma is well isotropized at relatively large scales that we consider.
}

\subsection{Applications}

One of our main applications is to study the structure, evolution and particle acceleration in non-relativistic collisionless shocks, which have long been considered as the most promising sites for producing CRs by means of the first-order Fermi acceleration mechanism (Fermi 1949; Bell 1978; Blandford \& Ostriker 1978). In particular, diffusive shock acceleration (DSA) of electrons and nuclei at supernova remnant (SNR) blast waves is expected to produce power-law energy distributions of accelerated particles extending over many energy decades (e.g. Morlino \& Caprioli 2012; Ackermann et al. 2013), which is thought to be responsible for most of the Galactic cosmic rays (CRs), possibly up to the "knee" part of the CR spectrum $\left(\sim 10^{15} \mathrm{eV}\right)$.

Theoretical investigation of CR acceleration in nonrelativistic collisionless shocks has a long history. Most earlier works either analytically or numerically solve the CR transport equations (e.g., Berezhko \& Volk 1997; Malkov 1997; Kang et al. 2002; Amato \& Blasi 2005; Zirakashvili \& Aharonian 2010; Caprioli 2012) or adopt a Monte-Carlo approach (e.g., Ellison et al. 1990, 1995; Niemiec \& Ostrowski 2004; Vladimirov et al. 2006). While these works generally yield results consistent with observations, they rely on quasi-linear theory for magnetic field amplification with simple prescriptions of CR diffusion coefficients and injection efficiency, reflecting the poorly known microphysics of particle injection and turbulence. In particular, the turbulence that is responsible for scattering CRs should be largely generated by the accelerated CRs themselves, which in turn affects the shock structure and the injection process. The complex interplay among these physical processes calls for selfconsistent numerical simulations.

PIC approach is at the frontier of this research. Simulations have been carried out using full PIC codes (e.g., Amano \& Hoshino 2007; Riquelme \& Spitkovsky 2011; Narayan et al. 2012; Guo et al. 2014), which mainly address electron acceleration, as well as hybrid-PIC codes (e.g., Quest 1988; Giacalone et al. 1992; Giacalone 2004; Gargaté \& Spitkovsky 2012; Guo \& Giacalone 2013), which mainly address ion acceleration. With growing computing power, simulations with relatively large domains and relatively long duration are becoming more affordable (e.g., Caprioli \& Spitkovsky 2013), allowing the physical processes such as particle injection, diffusion and acceleration efficiency to be studied in greater detail (Caprioli \& Spitkovsky 2014a,b,c). However, despite the successes of these state-of-the-art simulations, they still capture only the initial stages of shock evolution and particle acceleration. Larger spatial scales and longer time evolution are needed as particles accelerate to higher energies to accommodate the interactions of these particles with turbulence. In addition, current hybrid-PIC codes deal only with non-relativistic particles. Continued acceleration would in reality lead to transition into the relativistic regime, giving a different power-law slope in particle energy spectrum according to the Fermi acceleration theory (from $f(E) \propto E^{-3 / 2}$ to $E^{-2}$ in the test particle limit). This transition can not be captured in hybridPIC simulations, and remains to be confirmed. More- 
over, since the total energy in CRs $\left(\int E f(E) d E\right)$ would diverge as $E \rightarrow \infty$, we expect the fraction of CR particles $\left(\int f(E) d E\right)$ to decrease with time as particles are accelerated to higher energies. This reduction is important for understanding the long-term evolution of collisionless shocks, and again is too computationally demanding to be addressed by using the hybrid-PIC approach.

Without the need to resolve plasma scales, our MHDPIC approach can substantially outperform hybrid-PIC codes, allowing us to conduct large simulations following the long-term evolution of non-relativistic collisionless shocks with self-consistent particle acceleration. On the other hand, since the gas and CRs are treated separately, the main disadvantage of the MHD-PIC approach (as well as similar approaches, e.g., Reville \& Bell 2013) is that injection of $\mathrm{CR}$ particles has to be handled with ad hoc prescriptions. However, this may be remedied by properly designing controlled experiments to calibrate the injection recipes with hybrid-PIC simulations, as we defer to future work. In this paper, as the first application of our code to study particle acceleration in shocks, we adopt a simple and easily controllable injection prescription to demonstrate the capability of our code to handle highly non-linear regime of CR-gas interaction. Even with such a simple prescription, we are able to reproduce most of the essential features of shock structure and particle acceleration as in hybrid-PIC simulations. Most interestingly, we have performed one simulation with unprecedented long duration which followed particle acceleration from non-relativistic to relativistic regimes.

In addition, our formalism is well suited to study the interaction between the CRs and the gas in the ISM, potentially providing microphysical input for studying CR propagation and transport in the Galaxy (Strong et al. 2007). In particular, the non-linear wave-particle interaction of propagating CRs (Kulsrud \& Pearce 1969) is expected to play an important role in self-confinement of CRs in the Galaxy. In addition, given the dynamical importance of (especially low-energy) CRs in the ISM, the back-reaction from CRs may also affect the cascade of MHD turbulence (e.g., Lazarian \& Beresnyak 2006), and in turn their own propagation and transport in the Galaxy.

This paper is organized as follows. We derive the formulation of the CR-gas system in Section 2. Implementation of this formulation to the Athena MHD code is briefly described in Section 3, with details and code tests provided in Appendices A and B. As a special application of our formulation, we revisit the linear dispersion relation of the Bell instability in Section 4 and highlight the relevance of the CR-induced Hall term. In Section 5, we describe the setup of shock simulations with a simple prescription of particle injection. Simulation results presented and analyzed in Sections 6 and 7, highlighting the code capabilities and transition of particle acceleration from non-relativistic to relativistic regimes. We summarize and conclude in Section 8.

\section{FORMULATION}

We consider the interaction between a thermal plasma (gas) and cosmic ray (CR) particles. The gas is fully ionized, non-relativistic, and is treated as a magnetized fluid. It consists of ions, which contain all the inertia, and massless electrons, which mainly conduct current. CR particles are relativistic and collisionless, so that they are only subject to the Lorentz force. We assume that CRs are particles with only one sign of charge (positive). While CRs do not interact with the gas directly, they provide indirect feedback via interactions with electromagnetic fields. In this section, we formulate the equations for CR-gas interaction. We rigorously derive the form of CR feedback to the gas in the MHD framework in Section 2.1, where CRs are treated as fluid, and in Section 2.2, we discuss the motion of CRs as individual particles.

\subsection{MHD Equations with CR Feedback}

The effect of collisionless CRs on the thermal gas can be well represented by the CR charge density $n_{\mathrm{CR}}$ and current density $\boldsymbol{J}_{\mathrm{CR}}$ (zeroth and first moments of momentum distribution, treated as a fluid). They are related by

$$
\boldsymbol{J}_{\mathrm{CR}}=n_{\mathrm{CR}} \boldsymbol{u}_{\mathrm{CR}},
$$

where $\boldsymbol{u}_{\mathrm{CR}}$ can be interpreted as the mean drift velocity of CR particles.

CRs are subject to the Lorentz force, and the force density they experience is

$$
\boldsymbol{F}_{\mathrm{CR}}=n_{\mathrm{CR}} \mathcal{E}+\frac{1}{c} \boldsymbol{J}_{\mathrm{CR}} \times \boldsymbol{B},
$$

where $\mathcal{E}$ and $\boldsymbol{B}$ are the electric and magnetic fields.

The total current density in the system comprises of contributions from the gas (ions and electrons) and the CRs

$$
\begin{aligned}
\boldsymbol{J}_{\mathrm{tot}}=\frac{c}{4 \pi} \nabla \times \boldsymbol{B} & =\boldsymbol{J}_{g}+\boldsymbol{J}_{\mathrm{CR}} \\
& =n_{i} \boldsymbol{v}_{i}+n_{e} \boldsymbol{v}_{e}+n_{\mathrm{CR}} \boldsymbol{u}_{\mathrm{CR}},
\end{aligned}
$$

where $n_{i}$ and $\boldsymbol{v}_{i}\left(n_{e}\right.$ and $\left.\boldsymbol{v}_{e}\right)$ are the charge density and mean velocity of the ions (electrons). We are interested in scales much larger than the Debye length, where charge neutrality applies,

$$
n_{i}+n_{e}+n_{\mathrm{CR}}=0 .
$$

Note that being charge densities, we have $n_{i}>0$ and $n_{e}<0$. While we generally consider $\mathrm{CR}$ ions $\left(n_{\mathrm{CR}}>\right.$ 0 ), our formulation is equally applicable to sufficiently energetic CR electrons (e.g., whose energy is higher than energy of thermal ions), thus $n_{\mathrm{CR}}$ can be either positive or negative.

In general, we expect $\left|n_{\mathrm{CR}}\right| \ll n_{i} \approx\left|n_{e}\right|$, and this is the regime where our formulation is applicable: essentially all electrons come from the gas, thus they are largely thermal and can be treated as a massless fluid, obeying the force-free condition

$$
n_{e}\left(\mathcal{E}+\frac{\boldsymbol{v}_{e}}{c} \times \boldsymbol{B}\right)-\nabla \cdot \mathrm{P}_{e}=0,
$$

where $\mathrm{P}_{e}$ is the electron pressure tensor. 
Combining the equations above, we arrive at the generalized Ohm's law in the presence of CRs:

$$
\begin{aligned}
\mathcal{E}= & -\frac{\boldsymbol{v}_{g}}{c} \times \boldsymbol{B}+\frac{1}{\left|n_{e}\right| c} \boldsymbol{J}_{\mathrm{tot}} \times \boldsymbol{B}-\frac{1}{\left|n_{e}\right|} \nabla \cdot \mathrm{P}_{e} \\
& -\frac{n_{\mathrm{CR}}}{\left|n_{e}\right|} \frac{\left(\boldsymbol{u}_{\mathrm{CR}}-\boldsymbol{v}_{g}\right)}{c} \times \boldsymbol{B},
\end{aligned}
$$

where $\boldsymbol{v}_{g} \equiv \boldsymbol{v}_{i}$ is the bulk velocity of the gas (ions). In the above, the first term is the normal inductive term in MHD, the second corresponds to the usual Hall term, and the third is the gradient of the electron pressure. The last term is a novel term due to the presence of CRs, which we call the CR-induced Hall term (hereafter, CR-Hall term). It describes the relative drift between CRs and the bulk of the plasma.

Both the Hall term and the electron pressure gradient term typically become important only on scales smaller than the ion inertial length $c / \omega_{p i}$, where $\omega_{p i}=$ $\left(4 \pi n_{i}^{2} / \rho\right)^{1 / 2}$ is the ion plasma frequency, and $\rho$ is the gas mass density. To see this, we compare the Hall term with the induction term, and the Hall term becomes important when $\boldsymbol{J}_{\text {tot }} / n_{e}$ is comparable or larger than the Alfvén velocity $v_{A}=B / \sqrt{4 \pi \rho}$. Replacing $J_{\text {tot }}$ by $c B / L_{H}$, we see that the Hall term dominates on scales

$$
L_{H} \lesssim \frac{c B}{\left|n_{e}\right| v_{A}} \approx \frac{c}{\omega_{p i}},
$$

where we have used $\left|n_{e}\right| \approx n_{i} \gg\left|n_{\mathrm{CR}}\right|$. Similarly, assuming that the electron pressure is comparable to the gas pressure (which again generally requires $\left|n_{e}\right| \approx n_{i}$ ), one can further replace $\nabla \cdot \mathrm{P}_{e}$ by $\rho c_{s}^{2} / L_{P}$, where $c_{s}$ is the sound speed. It is straightforward to show that the electron pressure gradient term dominates the inductive term when

$$
L_{P} \lesssim\left(\frac{c_{s}^{2}}{v_{A}^{2}}\right)\left(\frac{c}{\omega_{p i}}\right)
$$

For typical plasmas where thermal and magnetic energies are in rough equipartition, we find $L_{P} \sim L_{H} \sim\left(c / \omega_{p i}\right)$.

Under the MHD framework, we are interested in scales much larger than $c / \omega_{p i}$, thus we ignore the conventional Hall and electron pressure gradient terms. We note that these two terms are explicitly included in the formulation for hybrid-PIC codes, which aim at resolving the physics on the scale of ion inertial length. In these codes, some prescription of the electron pressure tensor (i.e., electron equation of state) is needed as a closure relation to account for the unresolved electron microphysics. At much larger scales, however, MHD assumption is expected to hold (as a closure relation), and we arrive at the ideal MHD Ohm's law with the corrections due to the CRHall term

$$
\mathcal{E}=-\frac{\boldsymbol{v}_{g}}{c} \times \boldsymbol{B}-\frac{n_{\mathrm{CR}}}{\left|n_{e}\right|} \frac{\left(\boldsymbol{u}_{\mathrm{CR}}-\boldsymbol{v}_{g}\right)}{c} \times \boldsymbol{B} .
$$

Note that unlike the conventional Hall term, the CR-Hall term does not provide a typical lengthscale above which it can be safely neglected, and should be always kept in the formulation.
More insight into the importance of CR-Hall term can be gained by rearranging the above equation in two ways. First, we define

$$
R \equiv \frac{n_{\mathrm{CR}}}{\left|n_{e}\right|}=\frac{n_{\mathrm{CR}}}{n_{i}+n_{\mathrm{CR}}},
$$

the ratio of the $\mathrm{CR}$ charge density to the total charge density (of the electrons). Note that our formulation requires that $R \ll 1$. Equation (9) is then equivalent to

$$
\mathcal{E}=-\left[(1-R) \boldsymbol{v}_{g}+R \boldsymbol{u}_{\mathrm{CR}}\right] \times \boldsymbol{B} / c .
$$

This equation has a clear physical meaning, namely, the advection velocity of the magnetic field is the chargedensity-weighted mean velocity of the composite gas-CR system. We can also see that the CR-Hall term becomes dynamically important when the following quantity,

$$
\Lambda \equiv \frac{R\left(\boldsymbol{u}_{\mathrm{CR}}-\boldsymbol{v}_{g}\right)}{v_{A}},
$$

approaches unity. While we expect $R \ll 1$, CRs may achieve highly super-Alfvénic drift velocities relative to the gas so that $\Lambda \gtrsim 1$. Physically, from Equations (3) and (4), this requires the electron-ion drift velocity in the thermal gas to be on the order of $v_{A}$ or larger. Note that the super-Alfvénic streaming between background electrons and ions may lead to plasma streaming instabilities at the electron scale, e.g., Weibel-like filamentation was found at about 10 electron skin depths in full-PIC simulations by Riquelme \& Spitkovsky (2009). These effects are not captured in either MHD or hybrid-PIC methods. While our formulation remains formally valid for $\Lambda>1$, one may need to bear in mind potential caveats of unresolved electron-scale effects.

Second, we note that the CR-Hall term closely resembles the Lorentz force experienced by CRs. To proceed, we define

$$
\mathcal{E}_{0} \equiv-\frac{\boldsymbol{v}_{g}}{c} \times \boldsymbol{B},
$$

as the electric field in ideal MHD without CRs. Substituting Equation (9) into Equation (2), we can re-express the overall Lorentz force felt by CRs as

$$
\boldsymbol{F}_{\mathrm{CR}}=(1-R)\left(n_{\mathrm{CR}} \mathcal{E}_{0}+\frac{1}{c} \boldsymbol{J}_{\mathrm{CR}} \times \boldsymbol{B}\right) .
$$

This is an explicit manifestation of the effect of electromagnetic feedback: the overall Lorentz force experienced by CRs as a fluid is reduced by a fraction $R$ compared with the case without the CR-Hall term. Plugging Equation (14) back to Equation (9), the electric field can be rewritten in a particularly simple form

$$
\mathcal{E}=\mathcal{E}_{0}-\frac{\boldsymbol{F}_{\mathrm{CR}}}{n_{g}},
$$

where $n_{g} \equiv n_{i}$ is the bulk charge density of the (ion) fluid.

So far we have focused on the electromagnetic feedback from the CRs, which determines the evolution of the magnetic field via the induction equation,

$$
\frac{\partial \boldsymbol{B}}{\partial t}=-c \nabla \times \mathcal{E}
$$


Below we discuss the rest of the MHD equations for the gas.

CRs streaming in the gas also exchange momentum with the gas. Due to charge-neutrality, the gas (ions and electrons) possesses the opposite charge density to CRs. Correspondingly, the gas experiences exactly the opposite electric force density as CRs in any local volume of the system, namely, $-n_{\mathrm{CR}} \mathcal{E}$. In addition, as in Equation (3), the total current density in the system has contributions from both CRs, $\boldsymbol{J}_{\mathrm{CR}}$, and the gas, $\boldsymbol{J}_{g}$. The magnetic force experienced by the gas is $\boldsymbol{J}_{g} \times \boldsymbol{B} / c$, while the ideal MHD equations adopt $(\nabla \times \boldsymbol{B}) \times \boldsymbol{B} / 4 \pi=\boldsymbol{J}_{\text {tot }} \times \boldsymbol{B} / c$. Therefore, we need to compensate for the difference given by $-\boldsymbol{J}_{\mathrm{CR}} \times \boldsymbol{B} / c$, which is exactly the opposite of the magnetic force experienced by CRs. Combining these two facts, we see that the momentum feedback from CRs on the gas is simply to "kick" the gas with the opposite of the Lorentz force $\boldsymbol{F}_{\mathrm{CR}}$ experienced by CRs. This is an explicit manifestation of Newton's third law which guarantees total momentum conservation.

The energy feedback from CRs can be understood as the work done by the external force (CR momentum feedback) per unit time, namely, $\boldsymbol{v}_{g} \cdot\left(-\boldsymbol{F}_{\mathrm{CR}}\right)$. Using previous equations (2), (11), (13), and (14), we can easily derive

$$
\begin{aligned}
\boldsymbol{v}_{g} \cdot \boldsymbol{F}_{\mathrm{CR}} & =(1-R) \boldsymbol{v}_{g} \cdot\left(n_{\mathrm{CR}} \mathcal{E}_{0}+\boldsymbol{J}_{\mathrm{CR}} \times \boldsymbol{B} / c\right) \\
& =(1-R) \boldsymbol{J}_{\mathrm{CR}} \cdot \mathcal{E}_{0}=\boldsymbol{J}_{\mathrm{CR}} \cdot \mathcal{E} \\
& =\boldsymbol{u}_{\mathrm{CR}} \cdot \boldsymbol{F}_{\mathrm{CR}} .
\end{aligned}
$$

We note that $\boldsymbol{u}_{\mathrm{CR}} \cdot \boldsymbol{F}_{\mathrm{CR}}$ can be understood as the rate of energy gain by CRs due to the work done by the Lorentz force. The above equality means that the same amount of energy is lost by the gas, which is an explicit manifestation of energy conservation of the composite gas and $\mathrm{CR}$ system. Moreover, the equality with $\boldsymbol{J}_{\mathrm{CR}} \cdot \mathcal{E}$ indicates that the energy exchange with the gas is achieved via Joule heating (or cooling).

Based on the discussions above, the remaining of the ideal MHD equations for the gas can be written as follows (in conservative form)

$$
\begin{gathered}
\frac{\partial \rho}{\partial t}+\nabla \cdot\left(\rho \boldsymbol{v}_{g}\right)=0 \\
\frac{\partial \rho \boldsymbol{v}_{g}}{\partial t}+\nabla \cdot\left(\rho \boldsymbol{v}_{g}^{T} \boldsymbol{v}_{g}-\frac{\boldsymbol{B}^{T} \boldsymbol{B}}{4 \pi}+P_{g}^{*} \mathrm{I}\right) \\
=-(1-R)\left(n_{\mathrm{CR}} \mathcal{E}_{0}+\boldsymbol{J}_{\mathrm{CR}} \times \boldsymbol{B} / c\right)=-\boldsymbol{F}_{\mathrm{CR}} \\
\frac{\partial E}{\partial t}+\nabla \cdot\left[\left(E+P^{*}\right) \boldsymbol{v}_{g}-\frac{\left(\boldsymbol{B} \cdot \boldsymbol{v}_{g}\right) \boldsymbol{B}}{4 \pi}+\frac{c}{4 \pi}\left(\mathcal{E}-\mathcal{E}_{0}\right) \times \boldsymbol{B}\right] \\
=-(1-R) \boldsymbol{J}_{\mathrm{CR}} \cdot \mathcal{E}_{0}=-\boldsymbol{u}_{\mathrm{CR}} \cdot \boldsymbol{F}_{\mathrm{CR}}
\end{gathered}
$$

where $P_{g}^{*} \equiv P_{g}+B^{2} / 8 \pi, \mathrm{I}$ is the identity tensor, and the total energy density is defined as

$$
E=\frac{P_{g}}{\gamma-1}+\frac{1}{2} \rho v_{g}^{2}+\frac{B^{2}}{8 \pi} .
$$

In the above, $\rho, P_{g}$ are gas density and pressure, and $\gamma$ is the adiabatic index. There is also a source term in the energy flux in the energy equation (20), which accounts for the correction to the ideal MHD Poynting flux $\left(c \mathcal{E}_{0} \times \boldsymbol{B} / 4 \pi\right)$ due to the CR-Hall term.

Note that the $\mathrm{CR}$ feedback source terms are generally proportional to $n_{\mathrm{CR}} \propto R$. The requirement of $R \ll 1$ guarantees that as long as our formulation remains valid, the source terms would never become stiff to significantly affect the overall evolution of the gas.

To summarize, we have formulated the set of MHD equations appropriate for studying the interaction between CRs and the thermal plasma. Our formulation properly describes the physics of the composite system on scales much larger than the ion inertial length, $c / \omega_{p i}$, and when the CR charge and current density satisfy $R \ll 1$. We highlight that the presence of streaming CRs introduces electromagnetic feedback in the form of a CR-Hall term to the induction equation characterized by parameter $\Lambda$ (Equation 12). This term is of the same order as the momentum and energy feedback from CRs, and becomes dynamically important when $\Lambda$ is not much less than 1 , with the potential caveat that uncaptured plasma instabilites at unresolved electron scales may arise when $\Lambda \gtrsim 1$.

\subsection{Particle Treatment of CRs}

We adopted Gaussian units in our formulation in Section 2.1, which contains factors of speed of light, $c$, in the expressions. This factor has no physical significance in non-relativistic MHD and can be absorbed by redefining the electric field and charge/current density as $c \mathcal{E} \rightarrow \mathcal{E}$ and $\boldsymbol{J} / c \rightarrow \boldsymbol{J}$. On the other hand, our CR particles can be fully relativistic. Therefore, we artificially specify the speed of light $\mathbb{C}$ for the $\mathrm{CR}$ particles. For consistency with MHD, the value of $\mathbb{C}$ must be chosen such that it is much larger than any characteristic MHD velocities. We still keep the irrelevant factor $c$ so as to retain consistency and to contrast with the factor $\mathbb{C} .^{4}$

For individual particle $j$, we use $\boldsymbol{u}_{j}$ to denote its velocity. We further define the vector component of its four-velocity to be

$$
\boldsymbol{v}_{j} \equiv \gamma_{j} \boldsymbol{u}_{j}
$$

where the Lorentz factor

$$
\gamma_{j}=\frac{\sqrt{\mathbb{C}^{2}+v_{j}^{2}}}{\mathbb{C}}=\frac{\mathbb{C}}{\sqrt{\mathbb{C}^{2}-u_{j}^{2}}} .
$$

We define CR particle kinetic energy $E_{k}$ as the difference between its total energy and rest mass energy:

$$
E_{k, j} \equiv\left(\gamma_{j}-1\right) \mathbb{C}^{2}=\frac{v_{j}^{2}}{\gamma_{j}+1},
$$

which reduces to standard expressions of particle energy in both non-relativistic and relativistic regimes.

\footnotetext{
${ }^{4}$ One may continue to use $c$ to specify particle speed of light. We prefer to introduce a different symbol for conceptual clarity: in the non-relativistic MHD framework, $\mathbb{C}$ is a freely-variable numerical parameter rather than a physical "constant."
} 
The particle equation of motion reads

$$
\frac{d \boldsymbol{v}_{j}}{d t}=\left(\frac{q}{m c}\right)_{j}\left(c \mathcal{E}+\boldsymbol{u}_{j} \times \boldsymbol{B}\right) .
$$

where $(q / m c)_{j}$ represents particle charge-to-mass ratio. For the electric field, instead of using its primitive expression (9), we use the alternative expression from Equation (15). It has the clear advantage that the correction from the CR-Hall term is proportional to the momentum feedback $\left(\boldsymbol{F}_{\mathrm{CR}}\right)$, and hence no extra computation is needed. Note that here, and essentially in all other occasions, the factor $c$ can be absorbed into the $(q / m c)$ factor. This factor incorporates the particle intrinsic properties and is the only physical parameter to distinguish different CR particle species.

\section{IMPLEMENTATION}

We have implemented the MHD formulation described in $\S 2$ together with kinetic CR particles in the Athena MHD code (Stone et al. 2008). Hereafter, we will refer to our gas-particle framework as the MHD-PIC.

The Athena MHD code uses higher-order Godunov methods with constrained transport technique to enforce divergence-free condition on the magnetic field. Two MHD integrators are implemented in Athena, namely the corner transport upwind (CTU, Gardiner \& Stone 2005, 2008) and the van-Leer (VL, Stone \& Gardiner 2009) integrators. Both integrators are dimensionally unsplit and second order accurate in time, with the CTU integrator being more accurate and less diffusive (but more complicated). We always use the CTU integrator in our MHD-PIC applications, while changing the framework for the VL integrator would be a trivial extension.

Lagrangian particles have been implemented in the Athena MHD code in the context of protoplanetary disks (Bai \& Stone 2010a). These particles represent solid bodies interacting with the gas via aerodynamic drag. Backreaction from the particles to the gas (feedback) are also properly implemented which is an essential ingredient to study the streaming instability (Youdin \& Goodman 2005) as a pathway to planetesimal formation (Johansen et al. 2009; Bai \& Stone 2010b). The particle integrator and feedback are combined with the MHD integrator using a predictor-corrector scheme, which achieves second-order accuracy for the composite system. Taking advantage of the existing infrastructure for solid particles with feedback, we briefly describe our implementation of the CR particles with feedback below, with more details provided in Appendix A.

We employ the standard second-order accurate triangular-shaped cloud (TSC) scheme based on quadratic spline (QS) interpolation (Birdsall \& Langdon 2005) for interpolation of grid quantities to particle locations and for depositing particle quantities back to the MHD grid. Standard relativistic Boris integrator is used to push the particles (Boris 1970). The particle integrator is combined with the MHD integrator with feedback added as source terms in a similar predictor-corrector scheme as in Bai \& Stone (2010a) to achieve second-order accuracy. Exact conservation of momentum and energy in the composite system of gas and CRs is achieved by construction under the Godunov framework. We have further implemented sub-cycling for CR particles to improve the code performance. In practice, we generally choose 5 particle steps per MHD step considering the balance between accuracy and efficiency.

We have tested all ingredients of our implementation including the Boris integrator, CR feedback, and particularly the CR-Hall effect with carefully designed test problems, which are described in Appendix B.

\section{LINEAR ANALYSIS OF THE BELL INSTABILITY}

Before proceeding to applications, we first consider the non-resonant CR streaming instability of Bell (2004), and point out the relevance of the CR-Hall term that was previously neglected. This instability occurs when the CR drift velocity exceeds the local Alfvén velocity, where right-handed circularly-polarized modes become unstable as the Lorentz force exceeds magnetic tension. This instability has unstable wavelengths much smaller than the Larmor radius of the CR particles, and its free energy comes from the relative streaming of gas and CRs.

The Bell instability is considered to play an important role in the upstream of high Mach number nonrelativistic collisionless shocks, such as SNR shocks. Accelerated CRs drift with respect to the upstream fluid at velocity $U_{s}$, which is of the order the shock velocity or higher and is much larger than $v_{A}$. Since the typical growth time is much shorter than the advection time in the CR-induced shock precursor, the instability typically enters its strongly non-linear stage; the preshock magnetic field may be effectively amplified, and the selfgenerated magnetic turbulence feeds back on CRs, enhancing their diffusion. Hybrid-PIC simulations have recently shown that such a non-linear interplay between CRs and magnetic fields shapes the large-scale structure of the shock, and promotes rapid acceleration of energetic particles (Caprioli \& Spitkovsky 2014a,b,c).

We note that the CR-Hall term was not included either in the original MHD derivation of the instability (Bell 2004), or in the kinetic approach by Amato \& Blasi (2009). Here we re-derive the dispersion relation that properly includes the CR-Hall term. Suppose the background gas is uniform with density $\rho_{0}$ and zero velocity $\boldsymbol{v}_{g}=0$ (upstream reference frame). Let $\boldsymbol{B}_{0}$ be the background magnetic field, and $\boldsymbol{J}_{\mathrm{CR}}=n_{\mathrm{CR}} \boldsymbol{U}_{s}$ be the external current density provided by the CR particles. Both of them are along the $\hat{x}$ direction. Now we consider incompressible perturbations in the gas, assuming all physical quantities to evolve as $\exp \mathrm{i}(k x-\omega t)$ (note that the wave vector $\boldsymbol{k}$ is also along the $\hat{x}$ direction) and use $\boldsymbol{u}, \boldsymbol{b}$ to denote first-order perturbations of gas velocity and magnetic field. For incompressible modes, both quantities are in the transverse direction. From the momentum equation (19) (or more explicitly see Equation of (1) of Bell 2004), we have

$$
\begin{aligned}
-\mathrm{i} \omega \rho_{0} & (\boldsymbol{u} \times \boldsymbol{k})=+\mathrm{i}\left(\boldsymbol{k} \cdot \boldsymbol{B}_{0}\right)(\boldsymbol{b} \times \boldsymbol{k}) \\
& -(1-R)\left[\left(\boldsymbol{J}_{\mathrm{CR}} \times \boldsymbol{b}\right)-n_{\mathrm{CR}}\left(\boldsymbol{u} \times \boldsymbol{B}_{0}\right)\right] \times \boldsymbol{k},
\end{aligned}
$$

where for conciseness we have ignored factors containing $c$ and $4 \pi$, which will be irrelevant to the results. Also note the factor $(1-R)$ that comes from Equation 
(14). The perturbation equation for the induction equation (16) reads

$$
-\omega \boldsymbol{b}=(1-R)\left(\boldsymbol{k} \cdot \boldsymbol{B}_{0}\right) \boldsymbol{u}-R\left(\boldsymbol{k} \cdot \boldsymbol{U}_{s}\right) \boldsymbol{b} .
$$

The above equation gives the relation between $\boldsymbol{b}$ and $\boldsymbol{u}$.

Combining the two equations, and noting that $\boldsymbol{k}, \boldsymbol{B}_{0}$ and $\boldsymbol{J}_{\mathrm{CR}}$ are parallel, we find after some algebra

$$
\begin{aligned}
& \mathrm{i}\left[\omega^{2}-\omega k R U_{s}-(1-R) k^{2} v_{A}^{2}\right](\boldsymbol{b} \times \hat{x}) \\
& +(1-R) \frac{k J_{\mathrm{CR}} v_{A}^{2}}{B_{0}}\left(1-\frac{\omega}{k U_{s}}\right) \boldsymbol{b}=0 .
\end{aligned}
$$

Note that all terms containing $R$ are due to the CRHall term, and the most significant modification lies in the term $\omega k R U_{s}$, which leads to a non-negligible shift in wave frequency. Our $\Lambda$-parameter defined in Equation (12) corresponds to $\Lambda=R U_{s} / v_{A}$.

We provide the full derivation of the dispersion relation in Appendix C. In the following, we consider the limit $R \ll 1$ with no constraints on $\Lambda$, and just quote the results from the full derivation (see Equation C6)

$$
\left(\tilde{\omega} \pm \epsilon k_{0} v_{A}\right)^{2}=f\left(k \pm \frac{1}{f} k_{0}\right)^{2} v_{A}^{2}-\left(k_{0} v_{A}\right)^{2}\left(\frac{1}{f}-\epsilon^{2}\right),
$$

where the two \pm signs must be taken to be the same, and we have defined

$$
\begin{gathered}
\tilde{\omega} \equiv \omega-(\Lambda / 2) v_{A} k, \quad k_{0} \equiv \frac{J_{\mathrm{CR}}}{2 B_{0}}, \\
f \equiv 1+(\Lambda / 2)^{2}, \quad \epsilon \equiv \frac{v_{A}}{U_{s}} .
\end{gathered}
$$

Note that for highly super-Alfvénic shocks, $\epsilon \ll 1$. When the CR-Hall term is not included or for $\Lambda \ll 1$, we have $f=1$, and the dispersion relation (29) reduces to Equation (7) of Bell (2004) (regime II, since we treat CRs as a fixed external current). In the general case, the system becomes unstable when $\epsilon^{2}<1 / f$. The most unstable mode corresponds to $k_{m}= \pm k_{0} / f$, with fastest growth rate of the order $k_{0} v_{A} / \sqrt{f}$.

Now we discuss the role of the CR-Hall term, setting $\epsilon=0$, valid for highly super-Alfvénic shocks. Our linear analysis of the Bell instability leads to qualitative changes in two aspects. First, the most unstable wavenumber $k_{m}$ is reduced by a factor of about $f$. Without the CR-Hall term, we have $k_{m}=k_{0} \propto J_{\mathrm{CR}} \propto \Lambda$, which increases with $J_{\mathrm{CR}}$ without bound. Including this term, and considering the limit $\Lambda \gg 1$ (hence $\left.f \sim\left(R U_{s} / 2 v_{A}\right)^{2}\right)$ we have

$$
k_{m}=k_{0} / f \sim \frac{2 J_{\mathrm{CR}} v_{A}^{2}}{B_{0} R^{2} U_{s}^{2}} \sim\left(\frac{c}{\omega_{p i}}\right)^{-1} \frac{2}{\Lambda} .
$$

Matching the two limits of $\Lambda \ll 1$ and $\Lambda \gg 1$, we see that as $J_{\mathrm{CR}}$ (hence $\Lambda$ ) increases from zero, $k_{m} \propto J_{\mathrm{CR}}$, and the most unstable wavelength decreases until it reaches the scale of ion inertial length, at about $\Lambda \sim 2$. Further increasing $J_{\mathrm{CR}}$ would increase the most unstable wavelength. Therefore, the most unstable mode never falls below the scale of $c / \omega_{p i}$. This result may have important consequences for the resonant scattering of CRs in the vicinity of the shock front. Second, the fastest growth rate $\sim k_{0} v_{A} / \sqrt{f}$, is reduced by a factor of about $\sqrt{f}$. Without the CR-Hall term, the fastest growth rate increases with $J_{\mathrm{CR}}$ without bound since $k_{0} \propto J_{\mathrm{CR}}$. Including this term, and again in the limit $\Lambda \gg 1$, we have

$$
\omega_{\max } \sim \frac{J_{\mathrm{CR}} v_{A}^{2}}{B_{0} R U_{s}} \sim \Omega_{c} .
$$

Therefore, the maximum growth rate saturates at $\Omega_{c} \equiv$ $q B_{0} / m_{i} c$, the ion cyclotron frequency of the background plasma.

We note that using full kinetic PIC simulations, Riquelme \& Spitkovsky (2009) showed that when $\Lambda \gtrsim 1$, the Bell instability can be overtaken by a Weibel-like filamentation instability, found earlier by Niemiec et al. (2008). Given the fact that the growth of the Bell mode saturates at $\Omega_{c}$ and migrates to larger scales at $\Lambda \gg 1$, it is not surprising that the Bell mode is overtaken by the filamentation mode, which may not be captured in our MHD-PIC formulation.

We will show in our shock simulations that $\Lambda$ can approach order unity in the vicinity of the shock front. While this already corresponds to the non-linear stage of the Bell instability, our derivations in the linear regime still provide a useful quantitative assessment on the importance of the CR-Hall effect on the gas dynamics in this region.

\section{PARTICLE ACCELERATION IN COLLISIONLESS SHOCKS: SIMULATION SETUP}

As introduced in Section 1.1, we conduct a proof-ofconcept study of the evolution and particle acceleration in non-relativistic collisionless shocks to demonstrate our code performance, where the non-linear development of the Bell instability in the shock upstream plays a vital role (e.g., Bell et al. 2013; Caprioli \& Spitkovsky 2014b).

The basic setup of the shock problem involves colliding a highly super-Alfvénic flow into a conducting and static wall (left boundary in the Figures). The shock forms instantaneously, propagating away from the wall. After the collision, the downstream gas has zero longitudinal velocity, hence the simulation takes place in the downstream frame. Let $\hat{x}$ denote the direction of the shock, and the conducting wall is located at $x=0$. The colliding gas (upstream) moves from the right $(x>0)$ toward the wall, with uniform density $\rho_{0}$, pressure $p_{0}$ and velocity $-v_{0} \hat{x}$. We consider parallel shocks with magnetic field along the $\hat{x}$ direction, whose strength is $B_{0}$. For convenience, we define the shock Alfvénic Mach number to be $M_{A} \equiv v_{0} / v_{A 0}$, where $v_{A 0}=B_{0} / \sqrt{\rho_{0}}$ is the background Alfvén velocity ${ }^{5}$. The angle between the background field and shock normal $(\hat{x})$ is denoted by $\theta$ which, together with $M_{A}$, are the main shock parameters (the sonic Mach number is comparable to $M_{A}$ ).

For the purposes of this paper, we only consider high Mach number, parallel shocks with $M_{A}=30$. For highly super-sonic and super-Alfvénic shock, we have

\footnotetext{
5 More strictly, shock Mach number is defined in the shock frame, our definition simply follows the convention of Caprioli \& Spitkovsky (2013).
} 
$\rho_{0} v_{0}^{2} \gg P_{0}+B_{0}^{2} / 2$, which leads to shock jump conditions (expressed in the downstream frame) under ideal MHD

$$
\begin{array}{ll}
\rho_{1}=r \rho_{0}, & P_{1}=\frac{r}{r-1} \rho_{0} v_{0}^{2} \\
B_{x 1}=B_{x 0}, & v_{s h}=v_{0} /(r-1) .
\end{array}
$$

where $r \equiv(\gamma+1) /(\gamma-1)$ is the shock compression ratio, $v_{s h}$ is the velocity of the shock in the simulation (downstream) frame. For adiabatic index $\gamma=5 / 3$, we have $r=4$ for strong shock as usual. We use subscripts " 0 " and " 1 " to denote upstream and downstream quantities measured in the simulation (downstream) frame, hence $v_{1}=0$ by definition. The shock converts the kinetic energy in the upstream gas into thermal energy. The gas temperature can be defined as $p / \rho$, hence $T_{0}=P_{0} / \rho_{0}$, while $T_{1}=v_{0}^{2} /(r-1)$ is independent of $T_{0}$ for strong shocks. We also define the shock kinetic energy $E_{s h} \equiv v_{0}^{2} / 2$, which characterizes the energy of downstream thermal particles (per unit mass) and will be used as a scale to normalize the energy of CR particles.

The natural units for length, time and velocity are the ion inertial length $c / \omega_{p i}=(q / m c)^{-1} \rho_{0}^{-1 / 2}$, ion cyclotron frequency $\Omega_{c 0}=(q / m c) B_{0}$, and the Alfvén velocity $v_{A 0}=B_{0} \rho_{0}^{-1 / 2}$. They are all set to 1 in code units, with $\rho_{0}=B_{0}=(q / m c)=1$. We also set $T_{0}=1$ (or $\left.P_{0}=1\right)$, which is irrelevant as long as $T_{0} \ll v_{0}^{2}$. We are mainly interested in highly super-Alfvénic shocks so that $v_{0} \gg v_{A 0}$ (we choose $v_{0}=30$ ). This means that in the downstream frame, the gyro-radius of a thermal particle around background field is much larger than $c / \omega_{p i}$, by a factor of $\sim M_{A}$. Initially, we focus on particle acceleration in the non-relativistic regime, and set the particle speed of light to $\mathbb{C}=10^{4} \gg v_{0}$ (so that particles are not able to achieve relativistic velocities within the duration of simulations). In addition, we also run one simulation with much reduced speed of light $\mathbb{C}=10 \sqrt{2} v_{0}=424.3$ (or equivalently larger physical shock velocity). In this case, the energy for non-relativistic to relativistic transition $E_{k \text {,trans }} \sim \mathbb{C}^{2} / 2$ is about $200 E_{s h}$, which is optimal for studying particle acceleration trasitioning to the relativistic regime.

Being a higher-order Godunov code, the MHD integrator in Athena works very well for shock capturing. With uniform and homogeneous upstream medium, the shock is captured with only 2-3 grid cells using the CTU integrator with third-order reconstruction. The microphysics underlying the sharp transition in the MHD shock is the efficient particle thermalization process, mediated by electromagnetic turbulence as a result of plasma instabilities on microscopic scales $\left(\lesssim c / \omega_{p i}\right)$. While such plasma micro-instabilities are not captured in the MHD framework, we expect the scattering of CR particles to be dominated by turbulence on larger scales in highly superAlfvénic turbulence, which is captured in the MHD-PIC approach when streaming instabilities such as the Bell instability are fully developed.

Here we describe our simple prescription for particle injection. In every time step, we keep track of the loca-
TABLE 1

LIST OF MAIN SHOCK SIMULATION RUNS

\begin{tabular}{c|cccc}
\hline \hline Run & $\begin{array}{c}\text { Domain size } \\
L_{x} \times L_{y}\left(c / \omega_{p i}\right)\end{array}$ & $\begin{array}{c}\text { resolution } \\
\left(c / \omega_{p i} \text { per cell }\right)\end{array}$ & $\eta$ & $\mathbb{C}$ \\
& $\left(1.2 \times 10^{5}\right) \times 3000$ & 12 & $10^{-3}$ & $10^{4}$ \\
R1 & $\left(1.2 \times 10^{5}\right) \times 3000$ & 12 & $2 \times 10^{-3}$ & $10^{4}$ \\
R2 & $\left(1.2 \times 10^{5}\right) \times 3000$ & 12 & $4 \times 10^{-3}$ & $10^{4}$ \\
R4 & $\left(1.2 \times 10^{5}\right) \times 3000$ & 6 & $2 \times 10^{-3}$ & $10^{4}$ \\
R2-hr & $\left(3.89 \times 10^{5}\right) \times 4800$ & 12 & $2 \times 10^{-3}$ & 424.3 \\
R2-REL & $(3.824$ \\
\hline \hline
\end{tabular}

Note: all simulations correspond to parallel shocks with Alfvénic Mach number $M_{A}=30$.

tion of the shock front $x_{s}$ by computing the transversely averaged profile of $v_{x}$ along $\hat{x}$. This $1 \mathrm{D}$ profile is further smoothed with a Gaussian kernel whose width is 4 grid cells. The shock front $x_{s}$ is identified as the location where $v_{x}$ is reduced by about $40 \%$ with respect to the upstream value. With $x_{s}$ identified, the amount of mass traversed by the shock can also be obtained. We inject a spatially uniform, isotropic distribution of CR particles at the shock front in the co-moving frame with the shock, whose energy is set to be $10 E_{s h}$ in this frame. The amount (mass fraction) of CR particles we inject is a small and fixed fraction $\eta$ of the amount of mass swept by the shock. Note that $E_{k}=10 E_{s h}$ is approximately the threshold energy for a particle to be considered as nonthermal and to participate in the DSA process (Caprioli \& Spitkovsky 2014a; Caprioli et al. 2015). In brief, $\eta$ is our only parameter for particle injection, which encapsulates our ignorance of the physics of particle injection.

Physically, the injected particles originate from the gas that is newly processed by the shock. While we do not capture the injection physics with the simple prescription, we do compensate for the mass, momentum and energy of injected particles by subtracting them off from the gas at each timestep over certain width at the downstream side of the shock ${ }^{6}$, so that the total mass, momentum and energy in the full system are conserved. For typical injection fraction $\eta=2 \times 10^{-3}$ (see below), because the energy of injected particles only amounts to a very small fraction of the shock energy $(\sim 3 \%)$, influences to shock dynamics from this procedure is hardly noticeable. Also, the simulation outcome is very insensitive to the detailed implementation of the compensation scheme.

In the simulations, before the Bell instability is fully developed, most of the injected particles travel freely into the shock upstream. These streaming particles, on the one hand, source the initial growth of the Bell instability, while on the other hand, they are not effectively scattered since they only experience unperturbed or very weakly perturbed magnetic field. Particles injected later experience fully developed turbulence from the Bell instability and are efficiently scattered to enter DSA. As a result, there is a transient flow of unperturbed CR particles traveling through the upstream medium (with approximately constant $\left.j_{\mathrm{CR}}\right)$. Since the growth rate of the Bell

6 We further define the location of the tail end of the shock $x_{t}$ based on the 1D smoothed pressure profile (where $P$ achieves $\left.2 P_{1} / 3\right)$. Compensation is performed largely between $x_{t}$ and $x_{s}$. 


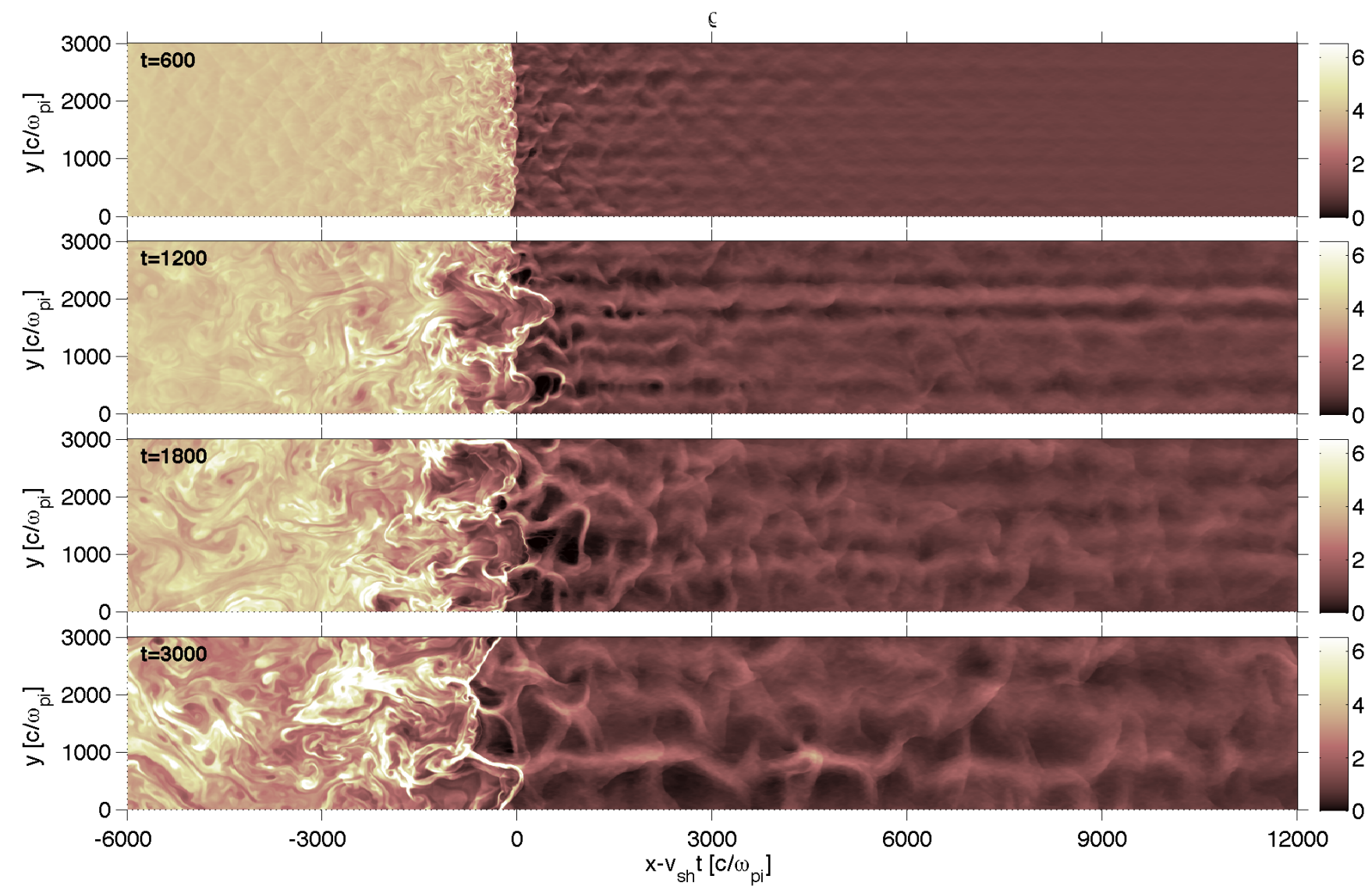

FIG. 1. - Snapshots of gas density at four different times (as indicated) from our fiducial run R2 which show the evolution of the shock. Only a small fraction of the simulation box is shown which is adjusted to cover the vicinity of the expected shock location at $x=v_{s h} t=v_{0} t / 3$.

instability is proportional to $j_{\mathrm{CR}}$, this transient CR flow would lead to growth of the Bell instability by a finite amplitude, as a result of the unrealistic initial condition. To remedy this artifact, we record the injection time $t_{i}$ for every CR particle. All particles with $t_{i}<480 \Omega_{c}^{-1}$ (which is more than sufficient for the Bell instability to be fully developed near the shock front) are removed at time $t=2 t_{i}$. Following this procedure, we expect our results to be largely unaffected by initial conditions at $t>960 \Omega_{c}^{-1}$.

We perform $2 \mathrm{D}$ shock simulations with fiducial domain size $L_{x}=1.2 \times 10^{5}\left(c / \omega_{p i}\right)$ and $L_{y}=3000\left(c / \omega_{p i}\right)$. We use conducting boundary condition at $x=0$, and inflow boundary condition at $x=L_{x}$, where all quantities are fixed at the initial value. With $M_{A}=30$, hence $v_{s h}=10$, we run the simulations up to time $t=3000 \Omega_{c 0}^{-1}$ so that at the end of the simulation, the gas initially at the right boundary just reaches the shock. We choose fiducial numerical resolution of $12 c / \omega_{p i}$ per cell, which will be shown to well capture the most unstable modes of the Bell instability. This is also much smaller than the Larmor radius of the lowest energy CR particles orbiting background field (for $E_{k}=10 E_{s h}$ particles, the Larmor radius $\left.R_{L} \sim 100 \mathrm{c} / \omega_{p i}\right)$. We also perform a resolution study with up to four times higher and two times lower resolution (see Section 6.4), while we mainly dis- cuss the run with twice resolution. Note that in hybridPIC simulations, the typical numerical resolution is 2 cells per $c / \omega_{p i}$ in order to properly capture the essential microphysics (e.g., Gargaté \& Spitkovsky 2012; Caprioli \& Spitkovsky 2013). With the MHD-PIC approach, the gain in efficiency is tremendous. ${ }^{7}$ For the simulation with reduced speed of light $\mathbb{C}=10 \sqrt{2} v_{0}=424.3$, we enlarge the domain size in both dimensions to $L_{x}=$ $3.888 \times 10^{5}\left(c / \omega_{p i}\right), L_{y}=4.8 \times 10^{3}\left(c / \omega_{p i}\right)$, and run the simulation to $t=11520 \Omega_{c 0}^{-1}$.

We choose $\eta=2 \times 10^{-3}$ as the standard CR mass fraction in our simulations. This is motivated by the numbers obtained from hybrid-PIC simulations (Caprioli \& Spitkovsky 2014a). In these simulations, it was found that in a parallel shock, about $\xi \approx 15 \%$ of the shock kinetic energy is converted to non-thermal particles. With $\eta=2 \times 10^{-3}$, the energy contained in our initially injected particles is about $3 \%$ of the shock energy. This is sufficiently small so as not to affect the shock structure, leaving enough room for these particles to further gain energy from the Fermi acceleration pro-

7 In addition, the particle timestep in our MHD-PIC simulations is mainly constrained by resolving the Larmor time and gridcrossing time, and with $\Upsilon=0.3$ (see Appendix A.4), the typical particle timestep $\Delta t>0.006 \Omega_{c 0}^{-1}$ is more than 6 times larger than used in hybrid-PIC. 
cess. Moreover, the most unstable wavelength of the Bell instability in the shock vicinity can be estimated to be $\lambda_{m} \sim 3 \pi /\left(\eta M_{A}\right) \sim 157\left(c / \omega_{p i}\right)$, which is well resolved with $\sim 13$ cells. Larger $\lambda_{m}$ is expected further upstream of the shock due to the reduction of net CR current density. As a parameter study, we also perform simulations with $\eta=10^{-3}$ and $\eta=4 \times 10^{-3}$. For all simulations, the number of particles we inject is equivalent to 4 particles per cell at background density $\rho_{0}$, or 16 particles per cell on average in the downstream. Note that despite the reduced spatial resolution, the number density of CR particles in physical units is comparable to that in typical hybrid-PIC simulations (where spatial resolution is much higher but only a very small fraction of the particles enters Fermi acceleration), and we have verified that simulation results converge with respect to number of injected particles. In the simulations, we allow a maximum of 5 particles steps per MHD step to speed up the calculation.

In sum, we mainly discuss 5 simulation runs: three runs with $\mathbb{C}=10^{4} \gg v_{0}$ using standard resolution with $\eta=10^{-3}, 2 \times 10^{-3}$ and $4 \times 10^{-3}$, one high resolution run, and one run with reduced speed of light $\mathbb{C}=10 \sqrt{2} v_{0}=424.3$. These runs are summarized in Table 1 , and each run is assigned a run name as indicated. We consider run R2 as the fiducial simulation and other runs as variations. All runs except for run R2-REL are relatively cheap computationally, with our fiducial run R2 taking about 2700 CPU hours on a HP Beowulf cluster with $3.47 \mathrm{GHz}$ Intel Westmere cores, yet the simulation box size and duration are already much larger than in the state-of-the-art hybrid-PIC simulations.

\section{PARTICLE ACCELERATION IN COLLISIONLESS} SHOCKS: NON-RELATIVISTIC REGIME

We focus on runs with $\mathbb{C} \gg v_{0}$ in this section, where all particles remain non-relativistic at all times. We begin by discussing simulation results from our fiducial run R2 in Sections 6.1 and 6.2, followed by parameter study in Section 6.3.

\subsection{Shock Evolution and Structure}

In our simulations, the Bell instability is first excited in the shock upstream due to the streaming CRs, and exhibits clear signature of circularly polarized modes in transverse magnetic and velocity fields, as expected from the linear eigenvector shown in Appendix B.2 (Equation B2). Growth into the non-linear stage leads to strong density variation and magnetic field amplification. In Figure 1, we show snapshots of gas density at four different times from our fiducial run R2. Note that only a small fraction $(15 \%)$ of the simulation box in the vicinity of the shock front is shown. While the Bell instability is essentially incompressible in the linear regime, the gas is gradually evacuated to produce cavities towards the nonlinear regime as a result of the filamentation instability (Bell 2005; Reville \& Bell 2012). The density structure found here is qualitatively very similar to that found in hybrid-PIC simulations of Caprioli \& Spitkovsky (2013), justifying the validity of our MHD-PIC approach. We note that over relatively long time evolution, the system develops larger and larger structures, which can only be accommodated with large transverse simulation domain size adopted in our simulations (as opposed to typical domain size of $\lesssim 1000 c / \omega_{p i}$ in hybrid-PIC simulations).

In Figure 2, we further show snapshots of the gas density and magnetic field strength at time $t=2400 \Omega_{c}^{-1}$ from all our simulations with non-relativistic particles (all runs except R2-REL). We focus on our fiducial run (second row) in this section. Accompanied by the Bell instability with cavitation and filamentation, we find that upstream magnetic fields are amplified by a factor of up to $\sim 3-5$ in the strongest regions (corresponding to density filaments), which is achieved gradually as the flow approaches the shock. Once processed by the shock, magnetic fields are amplified further due to compression and vorticity generation from the shock interface (e.g., Richtmeyer-Meshkov instability), and develop into filamentary structures of strongly enhanced fields a factor of up to 30 times the background field strength. All these features are very similar to results from self-consistent hybrid-PIC simulations of Caprioli \& Spitkovsky (2013).

Figure 3 further shows the profile of mean field strength in the vicinity of the shock front, where we have smoothed the profiles by averaging from time $t=$ $2300 \Omega_{c}^{-1}$ to $t=2500 \Omega_{c}^{-1}$ (with proper positional shift to account for shock motion). Focusing on run R2 here, we see that prior to the shock front, the mean field strength has increased by a factor of $\sim 2$. The downstream mean field strength is about $6-10$ stronger than the initial field $B_{0}=1$, which is again consistent with hybrid-PIC simulations of Caprioli \& Spitkovsky (2014a). We also note that while the downstream profile of mean field strength is highly time variable, which is also reflected in its large spatial variations, the profile shown in the Figure is rather typical.

We next show, in the upper panel of Figure 4, the transversely averaged profile of longitudinal CR current density $j_{\mathrm{CR}, x}$. It is measured in the co-moving frame of the gas (based on transversely averaged gas velocity in $\hat{x}$ ), and is the source of free energy in the Bell instability. The measured value of $j_{\mathrm{CR}, x}$ slowly decreases towards the far upstream of the shock to the level of $<0.01$ in code units, a factor of 8 smaller than the initial injected CR current density $(4 / 3) M_{A} \eta \sim 0.08$. This is because most of the particles are scattered back downstream within a short distance (diffusion length) from the shock front, and the far-upstream CR current is carried by escaping particles accelerated to much higher energies whose number density is much smaller (see the top panels of Figure 5 discussed in the next subsection for more information). These features are all consistent with hybrid-PIC simulations (Caprioli \& Spitkovsky 2014b). We can infer the most unstable wavelength for the Bell instability away from the shock front to be $\lambda_{m} \sim 4 \pi B_{0} / j_{\mathrm{CR}, x} \sim 1.2 \times 10^{3}\left(c / \omega_{p i}\right)$, which is very well resolved in our simulations. This scale is also consistent with the typical scale of density and magnetic fluctuations in the shock upstream present in Figure 2. Toward the shock front, the value of $j_{\mathrm{CR}, x}$ increases to its peak value of near 0.1 , which can be considered as the sum of injected CR current and the current from high energy CRs. It drops quickly to about zero in the down- 

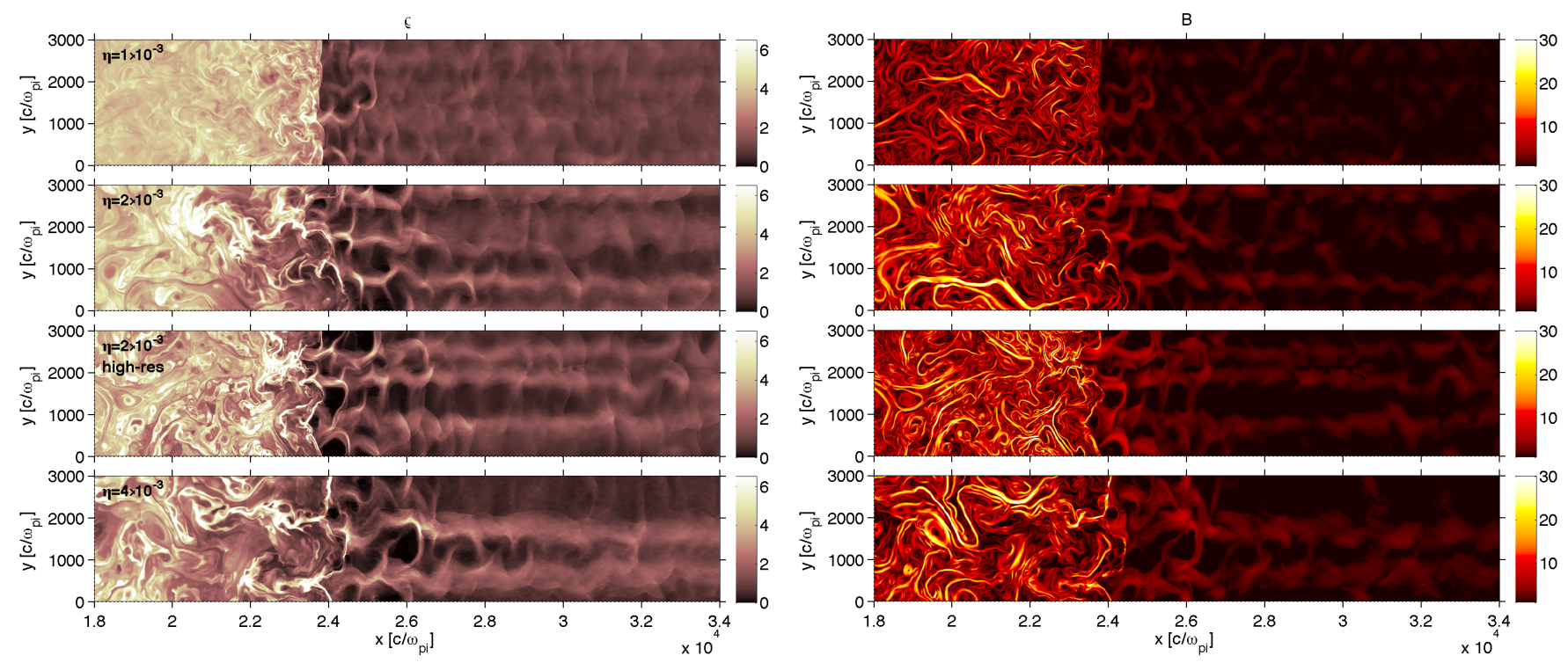

FIG. 2. - Simulation snapshots of gas density (left) and total magnetic field strength (right) at $t=2400 \Omega_{c}^{-1}$ from four of our simulation runs R1, R2, R2-hr and R4 with increasing injection efficiency $\eta$ as labeled in each panel. The third panels from top correspond to the high resolution run. The shock is approximately located at $x=24000$.

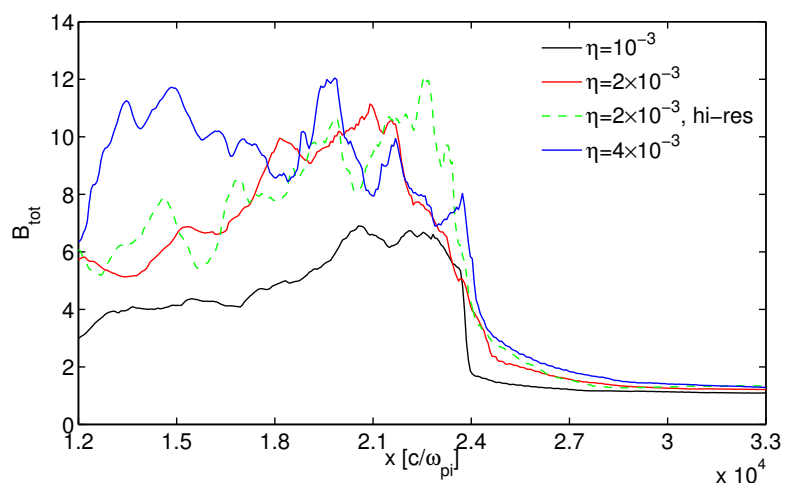

FIG. 3.- Transversely averaged profiles of magnetic field strength in the vicinity of the shock front around time $t=2400 \Omega_{c}^{-1}$ for four of our simulation runs R1, R2, R2-hr and R4.
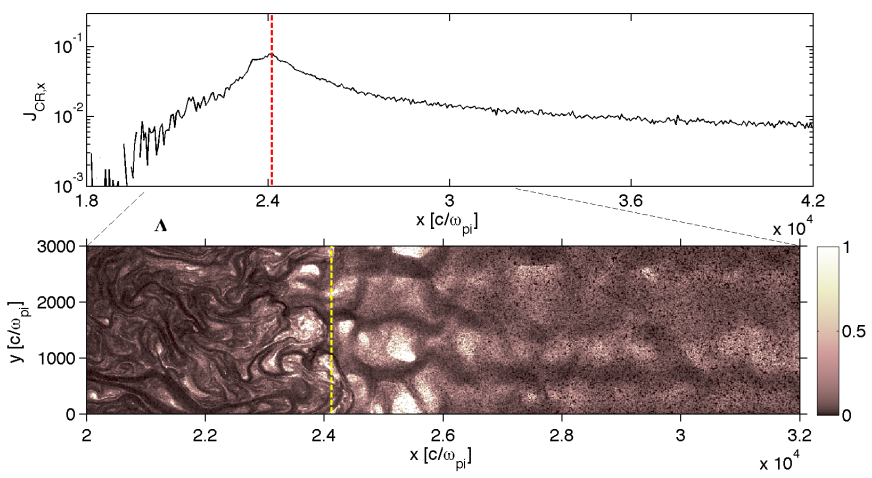

FIG. 4.- Results from our fiducial simulation R2 at $t=2400 \Omega_{c}^{-1}$. Top: transversely averaged $x$-component of the CR current density profile along $\hat{x}$ in the co-moving frame. Bottom: snapshot of $\Lambda$ defined in Equation (12) at $t=2400 \Omega_{c}^{-1}$, which measures the importance of the CR-Hall term. Vertical dashed lines in the two panels indicate the location of the shock front. stream, since no relative motion between gas and CRs is expected.

In Sections 2 and 4, we have highlighted the potential importance of the CR-Hall term and its relevance to the Bell instability in collisionless shocks. In the bottom panel of Figure 4, we further show the map of $\Lambda$ defined in Equation (12) at $t=2400 \Omega_{c}^{-1}$, which measures the importance of the CR-Hall term. The spatial distribution of $\Lambda$ is very non-uniform. Comparing with the corresponding panels in Figure 2, we see that $\Lambda$ is the largest in the density cavities, and achieves order unity near the shock front. We also see that at up to $\sim 10^{3}$ ion inertial lengths ahead of the shock, $\Lambda$ already has non-negligible deviations from zero. This result justifies the necessity of including the CR-Hall term to capture the role of streaming CRs on the evolution of magnetic fields. On the other hand, since $\Lambda \propto j_{\mathrm{CR}, x}$ by definition, we have $\Lambda \ll 1$ toward the far upstream (not shown in the Figure), and the CR-Hall effect becomes negligible.

\subsection{Particle Acceleration}

Since we inject particles with sufficiently high energy $E_{k}=10 E_{s h}$ into the shock upstream, most of the injected CR particles directly enter the DSA process. As long as the scattering process is isotropic in the comoving frame, the expected spectrum of accelerated particles does not depend on the details of the scattering, but only on the compression ratio $r$, with the momentum spectrum of accelerated particles to be $f(p) \propto p^{-q}$, where $q=3 r /(r-1) \simeq 4$ for $r \simeq 4$. The energy spectrum is related to the momentum spectrum via

$$
f(E)=4 \pi p^{2} f(p) \frac{d p}{d E} .
$$

For non-relativistic particles considered here, the energy spectrum is expected to have the form $f(E) \propto E^{-3 / 2}$.

In the bottom panel of Figure 5, we show the time evolution of the downstream particle energy spectrum 


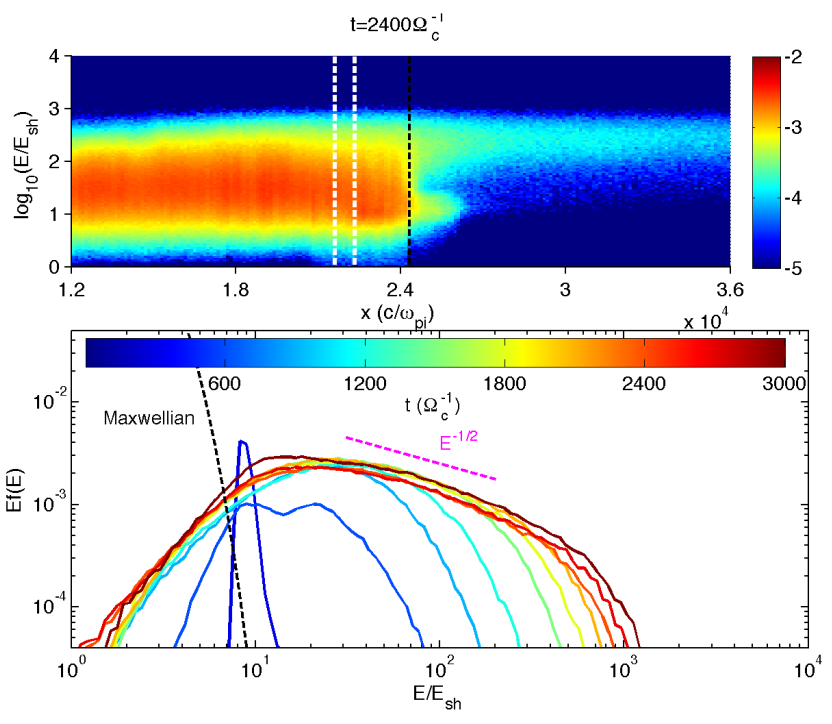

FIG. 5. - Energy spectrum (in dimensionless form $E f(E)$ ) of the CR particles from our fiducial run R2. The top panel shows the energy spectrum as a function of $x$ in units of $E_{s h}=v_{0}^{2} / 2$ at time $t=2400 \Omega_{c}^{-1}$. The bottom panel shows the time evolution of the downstream particle energy spectrum, as marked with different colors indicated by the color table. The spectrum is extracted by averaging through a layer at a fixed distance behind the shock front. As an example, vertical black dashed lines in the top panels mark the location of the shock front, and the vertical white dashed lines mark the layer where the downstream spectrum is extracted. The black dashed line shows the thermal energy spectrum with temperature $T=0.85 T_{1}$.

for our fiducial run R2. At early times before turbulence is fully developed $\left(\lesssim 300 \Omega_{c}^{-1}\right)$, the particle spectrum reflects the initial injected energy distribution, which peaks at $E=10 E_{s h}$. The spectrum then significantly broadens, extending substantially to the high energy side with a very small fraction scattered to low energy side. A highenergy power-law tail is gradually built up, with spectral slope consistent with $-3 / 2$. The high-energy tail extends to higher and higher energies with time, with a roughly exponential energy cutoff. We note that the normalization of the downstream spectrum appears to vary slightly in time and space, which reflects the stochasticity of the shock. For example, by performing additional simulations with different random seeds, we quote a relative uncertainty of $\sim 20 \%$ in the normalization.

We also show in black dashed line the expected energy spectrum for a thermal particle distribution (Maxwellian) in the downstream

$$
E f(E)=4 \times(2 / \sqrt{\pi})\left(E / E_{\mathrm{th}}\right)^{3 / 2} e^{-E / E_{\mathrm{th}}},
$$

where we choose $E_{\text {th }}=0.85 T_{1}$ (note that $T_{1}=v_{0}^{2} / 3$ is the downstream temperature from the shock jump condition). The deduced $15 \%$ serves as a proxy for the fraction of kinetic energy converted to accelerate particles, and the pre-factor of 4 is to account for shock compression. Clearly, the Maxwellian distribution is distinct from our CR energy distribution as they can not be smoothly joined together. In reality, as shown in self-consistent hybrid-PIC simulations, the energy spectrum is smooth across the entire energy range thanks to a population of supra-thermal particles whose energy lies between a few
$E_{s h}$ to about $10 E_{s h}$ (Caprioli \& Spitkovsky 2013). The presence of the supra-thermal particles is related to the particle injection process that bridges the gap between thermal and non-thermal particle populations (Caprioli et al. 2015). While this piece of physics is currently missing in our simple injection prescription, our current prescription may serve as a first approximation to mimic the injection process, with injected $E=10 E_{s h}$ particles presumably fed by the supra thermal particle population. We plan to develop and employ more realistic injection prescriptions in the near future.

In the upper panel of Figure 5, we show the spatial distribution of the particle energy spectrum at relatively late stage of the shock with $t=2400 \Omega_{c}^{-1}$. We see that high-energy CRs with energies $\sim 100 E_{s h}$ or higher penetrate into the shock upstream and provide the source of CR current to drive the Bell instability. The lower protrusion at $E_{k} \sim 10 E_{s h}$ into the shock upstream is mainly due to the artificial injection procedure adopted here, and we see that the initially injected CR particles are mostly confined in regions close to the shock front. We measure the particle energy spectrum at a fixed distance of about $2400 \mathrm{c} / \omega_{p i}$ behind the shock, as indicated in the Figure. As discussed in Caprioli \& Spitkovsky (2014a), this allows the shape of the particle energy spectrum to be fully settled.

We have also examined the evolution of the maximum particle energy. While we can always identify the particle with the maximum energy, the time evolution of this energy can be rather noisy due to random scattering and due to rapid changes when such particle leaves the domain. Here, we instead consider the following definition based on the full energy spectrum

$$
\bar{E}_{\max }=\frac{\int E^{n+1} f(E) d E}{\int E^{n} f(E) d E},
$$

where $n$ is an integer number of our choice. Note that if the energy distribution function takes the form $f(E) \propto E^{-m} \exp \left(-E / E_{\text {cut }}\right)$, then this integral yields $\bar{E}_{\text {max }} \approx(n+1-m) E_{\text {cut }}$. Empirically, we choose $n=6$, which corresponds to $\bar{E}_{\max }$ being $\sim 5-5.5 E_{\text {cut }}$ if the cutoff is exponential. We also find that the number obtained this way is approximately half the absolutely maximum particle energy.

The time evolution of $\bar{E}_{\max }$ is directly related to the rate of particle diffusion across the shock, and hence closely probes the properties of the MHD turbulence selfgenerated by streaming CRs. This is shown in Figure 6 . Excluding the time before $t=960 \Omega_{c 0}^{-1}$ (which is expected to be affected by initial conditions), we see that $\bar{E}_{\max }$ increases approximately linearly with time. This is consistent with efficient turbulent diffusion/scattering of particles with diffusion coefficient $D_{p} \propto v_{p} R_{L}$, where $v_{p}$ and $R_{L}$ are particle velocity and gyro-radius (Caprioli \& Spitkovsky 2014c). Being a proof-of-concept study with simplified injection prescription, performing more quantitative analysis on the properties of magnetic turbulence and particle diffusion is beyond the scope of this work, but we expect the detailed analysis performed in Caprioli \& Spitkovsky (2014b,c) to hold in general.

Based on the energy spectrum, we can measure the ef- 


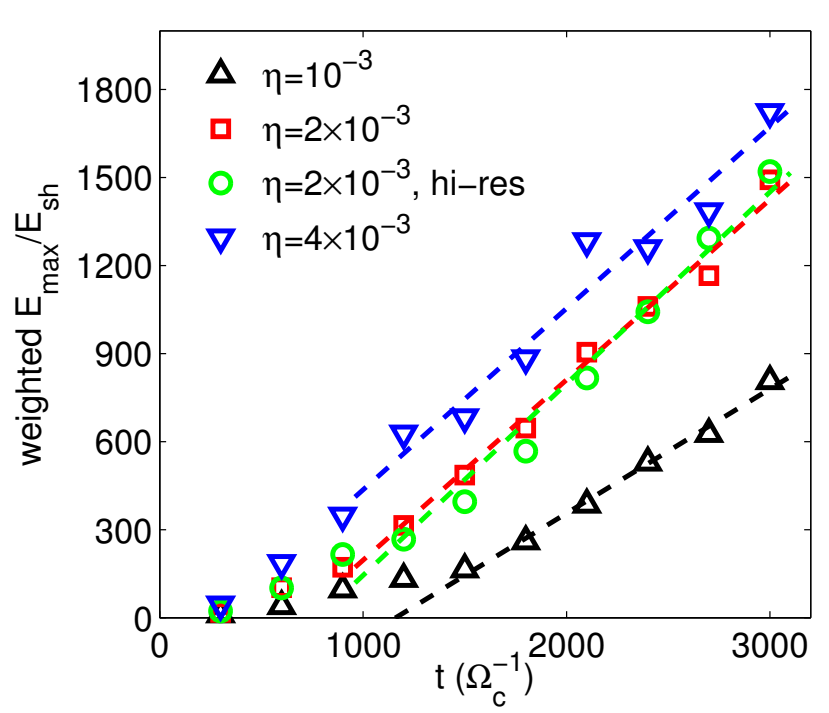

FIG. 6. - Time evolution of the weighted maximum particle energy $\bar{E}_{\text {max }}$ from four of our simulation runs R1, R2, R2-hr and R4 with increasing CR injection efficiency $\eta$. Also shown are linear fits to the data with $t>960 \Omega_{c 0}^{-1}$.

ficiency of particle acceleration $\xi$ by calculating the fractional energy residing in the CR particles compared with the shock kinetic energy $\left(m v_{s h}^{2} / 2\right)$. In the simulations, we find that this fraction first increases with time, and then achieves an approximately constant level but still varies with time at some level. By averaging the measured efficiency over the last $500 \Omega_{c}^{-1}$ of our simulations, we find $\xi \approx 13 \%$ from our fiducial run $\mathrm{R} 2$ (with relative uncertainty of $\sim 20 \%$, as discussed earlier), which is comparable to the efficiency obtained in hybrid simulations (Caprioli \& Spitkovsky 2014a).

\subsection{Parameter Dependence}

The outcome of the simulations mainly depends on the prescribed CR injection fraction $\eta$, an artificial parameter of the simulations. Snapshots of the shock structure for all simulations with non-relativistic particles at $t=2400 \Omega_{c 0}^{-1}$ are shown in Figure 2. For relatively small injection fraction $\eta=10^{-3}$ (run R1), the CR current streaming through the upstream, and hence the growth rate of the CR-driven instabilities, are relatively small. Correspondingly, it takes longer for cavitation and filamentation to develop, with smaller density contrast before reaching the shock front. While turbulence is generated in the upstream, the shock profile remains sharp at all times in our simulation.

When increasing $\eta$, cavitation becomes stronger, leading to higher density contrast and stronger magnetic fluctuations in the shock upstream. As a result, the shock front is more disturbed, leading to thicker shock transition layer (which is more evident in the temperature profile not shown in the plots). For run R4 with $\eta=4 \times 10^{-3}$, violent turbulence already dominates the shock near upstream, and the shock front substantially smoothed but is still identifiable. We have also performed simulations with $\eta=5 \times 10^{-3}$, and find that the shock front is so much disrupted that it becomes difficult to identify the

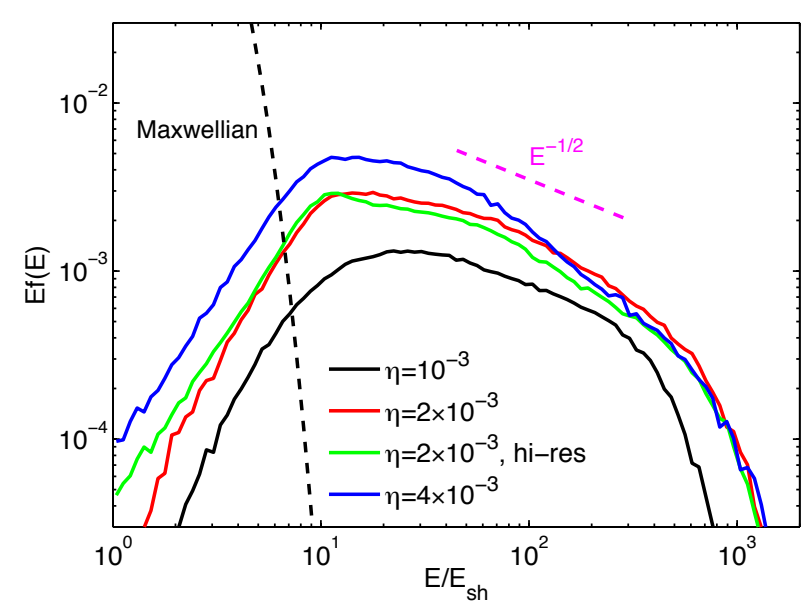

FIG. 7.- CR particle energy spectrum at $t=3000 \Omega_{c}^{-1}$ in the shock downstream from our four simulation runs R1, R2, R2-hr and R4. The black dashed line shows the thermal energy spectrum with temperature $T=0.85 T_{1}$.

location of the shock. In realistic shocks, such strong modification of shock structure is expected to lead to a reduction of the injection efficiency, and we conclude that imposing a constant $\eta \gtrsim 4 \times 10^{-3}$ leads to an unphysical over-injection.

Different levels of magnetic field amplification in the shock upstream can be clearly identified in Figure 3, where the mean field strength near the shock front on the upstream side increases with increasing $\eta$, as a result of stronger upstream CR current. Correspondingly, the downstream field strength also increases with $\eta$ within the duration of our simulations.

Particle acceleration is very efficient in all simulations. Figure 7 shows the cross-comparison of downstream particle energy spectrum measured at $t=3000 \Omega_{c}^{-1}$, measured from our four simulations with increasing $\eta$. For $\eta \lesssim 2 \times 10^{-3}$, the particle energy spectra follow the $f(E) \propto E^{-3 / 2}$ profile before the cutoff, while with relatively large $\eta=4 \times 10^{-3}$, the spectra slope is less consistent, especially at higher energies. Clearly, the total energy contained in the CR particles increases with the injection parameter $\eta$, and we quote $6 \%$ and $20 \%$ as the acceleration efficiency $\xi$ from runs with $\eta=10^{-3}$ and $4 \times 10^{-3}$, respectively. The former is smaller than obtained from hybrid-PIC simulations, indicating underinjection, while the latter is higher, indicating overinjection. The deviation from the expected spectral shape in run R4 may be the result of over-injection.

In all cases, the maximum particle energy increases approximately linearly with time, as seen from Figure 6 . The rate of increase is shallower with smaller $\eta$, suggesting less efficient particle diffusion. This is in line with the fact that there is less magnetic field amplification/fluctuation with smaller $\eta$, which leads to larger diffusion coefficient. Increasing $\eta$ to $4 \times 10^{-3}$, the rate at which $\bar{E}_{\text {max }}$ grows follows more closely with our fiducial run, and also shows larger fluctuations. This also reflects the saturation of magnetic field amplification and particle acceleration efficiency, again suggestive of overinjection. 
Overall, our parameter study suggests that the sharpness/smoothness of the shock depends on the prescribed particle injection efficiency $\eta$, which also determines the efficiency of particle acceleration $\xi$. Our fiducial choice of $\eta=2 \times 10^{-3}$ appears to yield results most consistent with hybrid-PIC simulations, at least within the duration of our runs. Over-injection of particles would over-smooth the shock. Since this is not observed in self-consistent simulations, particle injection must be suppressed when the shock transition layer is over-smoothed, so that in reality the shock structure remains reasonably sharp, and the particle acceleration efficiency should be kept to be within a certain level $\lesssim 20 \%$. In this sense, we conclude that particle injection must be a self-limiting process.

\subsection{Convergence}

Our high-resolution run R2-hr is in most aspects very similar to our fiducial run R2. Comparing their shock structures in Figure 2, we see that run R2-hr develops cavities at similar locations relative to the shock front as the run R2. The density contrast in these cavities and the level of magnetic field amplification are also similar between the two runs. While the shock transition region in the high resolution run appears to be thinner than in the low resolution run at this particular snapshot, this is mostly due to the very dynamic nature of the shock with highly chaotic turbulence. Over longer timescales, the two runs show no significant difference in shock appearance. More definitive evidence of convergence is revealed in Figures 6 and 7, where we see that the particle energy spectrum (hence acceleration efficiency), together with the evolution of maximum particle energy, agree quantitatively between the low and high resolution runs. This in turn implies that the particle diffusion coefficients are quantitatively similar between the runs.

We have also computed the spectral energy distribution $\mathcal{F}\left(k_{x}\right)$ of transverse magnetic fluctuations following the approach of Caprioli \& Spitkovsky (2014b) (see their Equation (3)), and show the results in Figure 8. Here, $\mathcal{F}\left(k_{x}\right)$ is dimensionless and measures the energy density of magnetic fluctuations per logarithmic wavenumber, normalized to background field energy density. To better demonstrate convergence, we performed two additional runs with half the resolution of $\mathrm{R} 2$ and twice the resolution of R2-hr, and hence in total we have four runs with resolution spanning from 24 to $3 c / \omega_{p i}$ per cell. We compute $\mathcal{F}\left(k_{x}\right)$ at fixed distances at the upstream and downstream sides of the shock for each run (see the caption of Figure 8 for details), averaged over time interval between 2160 and $2400 \Omega_{c}^{-1}$.

We see that in the upstream, the Bell instability is well resolved at all resolutions (modulo uncertainties in the overall normalization as discussed previously). In all cases, the spectrum peaks at $k_{x} \sim 0.008\left(c / \omega_{p i}\right)^{-1}$, corresponding to the fastest growing Bell mode ahead of the $\operatorname{shock}^{8}$. In the downstream, the lowest resolution simulation have somewhat less power and may be under-resolved (but can also be due to larger noise and

\footnotetext{
8 This corresponds to longer wavelength than the naive estimate in Section 5, since most injected particle are scattered back shortly without reaching large distance into the upstream.
}

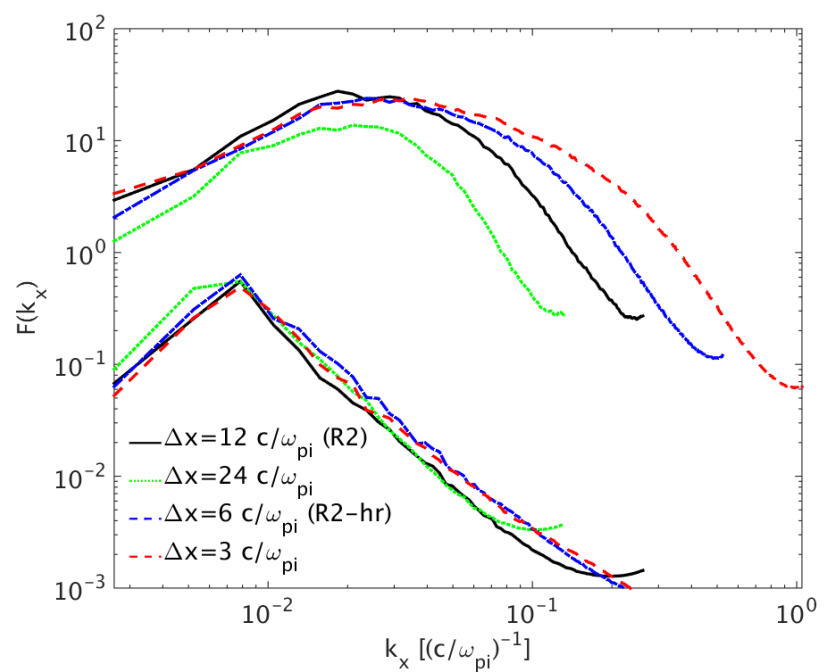

FIG. 8.- Dimensionless spectral energy distribution $\mathcal{F}\left(k_{x}\right)$ of transverse magnetic fluctuations from our resolution study of shock simulations, averaged over a time period between 2160 and 2400 $\Omega_{c}^{-1}$. Upper lines correspond to downstream spectrum, computed over a distance of $2400 \mathrm{c} / \omega_{p i}$ in $x$ starting from $2000 \mathrm{c} / \omega_{p i}$ behind the shock. Lower lines correspond to upstream spectrum, computed over a distance of $2400 \mathrm{c} / \omega_{p i}$ in $x$ starting from $4000 \mathrm{c} / \omega_{p i}$ ahead of the shock. Different colors correspond to different resolutions, as indicated by the legend, where black solid lines correspond to our fiducial resolution run R2. The rightmost point of each line corresponds to grid scale of the respective runs.

variability since there are fewer resolution elements compared to higher resolution runs). For our fiducial run R2, the power spectrum matches well with higher resolution runs at small $k_{x} \cdot{ }^{9}$ Increasing resolution, the spectrum extends to higher $k_{x}$. We note that for typical grid-based MHD code, $\sim 16$ cells are required to properly resolve a single wave mode without strong numerical dissipation (e.g., see Figures 7 and 12 of Stone et al. 2008 and discussions therein). This is exactly the scale where the power spectrum of our fiducial run R2 starts to deviate from higher-resolution runs at $k_{x} \sim 0.03\left(c / \omega_{p}\right)^{-1}$. We also see from the highest resolution run that interestingly, the downstream spectrum peaks at a scale about four times smaller than the scale of the upstream spectral peak. This may be simply understood as a result of shock compression.

Overall, since $\mathrm{CR}$ acceleration relies on the turbulent power at larger scales, which are well resolved in all runs, this leads to consistent calculation of the particle spectrum and of the maximum energy achieved by accelerated particles (see Figures 6 and 7). Our convergence study thus gives us confidence that our fiducial resolution of $12\left(c / \omega_{p i}\right)$ per cell is sufficient to capture the essential physics.

\section{PARTICLE ACCELERATION IN COLLISIONLESS SHOCKS: TRANSITION TO RELATIVISTIC REGIME}

In this section, we discuss our last run R2-REL, where we use a reduced particle speed of light $\mathbb{C}=10 \sqrt{2} v_{0}$

9 While the spectra do not line up exactly at lowest $k_{x}$, this is most likely due to small number statistics of long-wavelength modes. 
to study particle acceleration with transition from nonrelativistic to relativistic regime. Note that the initial particle energy of $E_{k}=10 E_{s h}$ corresponds to typical particle velocity of $v \sim 3.2 v_{0} \sim 0.22 \mathbb{C}$. The corresponding $\gamma \approx 1.025 \approx 1$, hence particles can still be considered as non-relativistic. Transition to the relativistic regime occurs approximately when $E_{k, \text { trans }} \sim \mathbb{C}^{2} / 2=200 E_{s h}$, where $\gamma \approx 1.5$. This way, we expect the particle energy spectrum to span nearly one decade before becoming relativistic. We run the simulations sufficiently long to achieve another decade of energy span in the relativistic regime ${ }^{10}$.

\subsection{Shock Structure and Evolution}

In Figure 9, we show the time evolution of the gas density in the vicinity of the shock front. While the overall sequence shares similarities with our fiducial run R2 shown in Figure 1, there are several points worth further discussion.

First, we clearly see that the waves and filamentation/cavity structure in the shock upstream progress to larger sizes over time. This is the main reason that we have enlarged our box size to $4800\left(c / \omega_{p i}\right)$ to accommodate the growing structures. Near the end of the run (bottom panel), the size of the largest structure is already a significant fraction of the transverse box size. Our additional test simulations with smaller transverse box size tend to saturate into a state with a single dominant transverse mode in the shock upstream, which may affect the scattering of energetic CR particles.

Second, we notice that the shock upstream in this run appears to be less perturbed and has a sharper shock transition layer compared to our fiducial run R2 (e.g., at time $\left.t=2400-3000 \Omega_{c}^{-1}\right)$. This is mainly due to the use of a smaller speed of light than in run R2. Note that the turbulence in the shock upstream is mainly excited by the escaping CRs, whose contribution is mainly due to the net CR current density. However, with a reduced speed of light, the CR velocity saturates at a smaller value of $\mathbb{C}$ : higher-energy $\mathrm{CR}$ particles become relativistic and do not contribute as much current as they do as non-relativistic particles. This effect becomes more pronounced with time, since the particles that penetrate into the shock upstream have higher energies.

Third, we notice that at later times the shock velocity slightly deviates from the standard jump condition of $v_{s h}=v_{0} / 3$, dropping by about $10 \%$ from time $t=4800 \Omega_{c}^{-1}$ to $10800 \Omega_{c}^{-1}$. As a result, the shock front lags from the expected position (Figure 9), and the shock compression ratio density is $r \approx 4.3$, slightly larger than expected from standard jump condition (Equation 34). This is a direct consequence of channeling of a sizable fraction of the upstream ram pressure into accelerated particles that do not feel the standard entropy jump at the shock, as discussed in $\S 6.2$ of Caprioli \& Spitkovsky (2014a). When relativistic CRs contain a sizable frac-

\footnotetext{
10 The time we terminate the simulation corresponds to the maximum time when the flow that reaches the shock front is unaffected by the boundary condition at the rightmost end of the box. Since it takes time for the accelerated particles to reach the right boundary, this time is larger than $3 L_{x} / 4 v_{0}=9720 \Omega_{c}^{-1}$.
}

tion of the energy density, a further increase of the compression ratio is expected because of the contribution of relativistic CR fluid (whose adiabatic index is $4 / 3$ ), but this effect is still negligible in the simulations presented here. We also find the development of a CR-induced precursor, in which the pre-shock fluid is slowed down and heated up because of the pressure in diffusing CRs and because of turbulent heating (see Section 6.1 of Caprioli \& Spitkovsky 2014a). Note that both the shock precursor and the increase of shock compression ratio are also observed in our previous non-relativistic runs.

\subsection{Particle Acceleration}

The CR spectrum at the end of the simulation together with its time evolution are shown in Figure 10. Since we expect the particle momentum spectrum $f(p) \propto p^{-4}$ to be a universal consequence of Fermi acceleration regardless of being in the non-relativistic or relativistic regimes, we show the time evolution of the particle momentum spectrum in the bottom panel of the Figure. Given our definition of particle kinetic energy $E_{k}$ from Equation (24), and using Equation (35), particle momentum spectrum is related to energy spectrum by

$$
4 \pi p^{3} f(p)=E f(E) \frac{\mathbb{C}^{2} p^{2}}{E\left(E+\mathbb{C}^{2}\right)} .
$$

We see that the momentum spectrum, plotted in dimensionless form $4 \pi p^{4} f(p) / v_{0}$, exhibits approximately flat shape both in the non-relativistic regime with $p / v_{0}<$ 14.2 , and in the relativistic regime toward higher momenta $p / v_{0} \gtrsim 20$, consistent with standard theory of Fermi acceleration. ${ }^{11}$ The relativistic part of the spectrum has slightly smaller normalization than the nonrelativistic part, while they connect smoothly through the transition. We also show the energy spectrum in the middle panel of the Figure. Note that for a momentum spectrum of $f(p) \propto p^{-4}$, the energy spectrum should not clearly transition to the $f(E) \propto E^{-2}$ scaling until Lorentz factor $\gamma \gtrsim 10\left(\sim 2000 E_{s h}\right)$. Since our CR energy spectrum already drops off at $E \gtrsim 1500 E_{s h}$, we do not yet cover the fully relativistic portion of the spectrum.

In the top panel of Figure 10, we see that CRs escaping into the far upstream near the end of the run almost completely consist of relativistic particles whose energy is well above $200 E_{s h}$. We also find that more contributions come from non-relativistic particles toward earlier times. These observations are consistent with the reduction of $j_{\mathrm{CR}, x}$ with time discussed earlier and is mainly responsible for the weaker turbulence in the shock upstream.

From the simulation, we find that the acceleration efficiency $\xi$, slowly increases at early time, and saturates to about $20 \%$ in the later half of the run. The relatively large acceleration efficiency is in line with the reduction of shock velocity discussed earlier. We also note that the value of $\xi$ in this run is about $15 \%$ at $t \sim 3000 \Omega_{c}^{-1}$, comparable to $13 \%$ from our fiducial run R2.

\footnotetext{
11 While we also expect the spectral slope to slightly deviate from standard value due to the change in shock compression ratio, the deviation is at the level of 0.1 , which is not distinguishable due to noise in the particle energy and momentum spectra.
} 


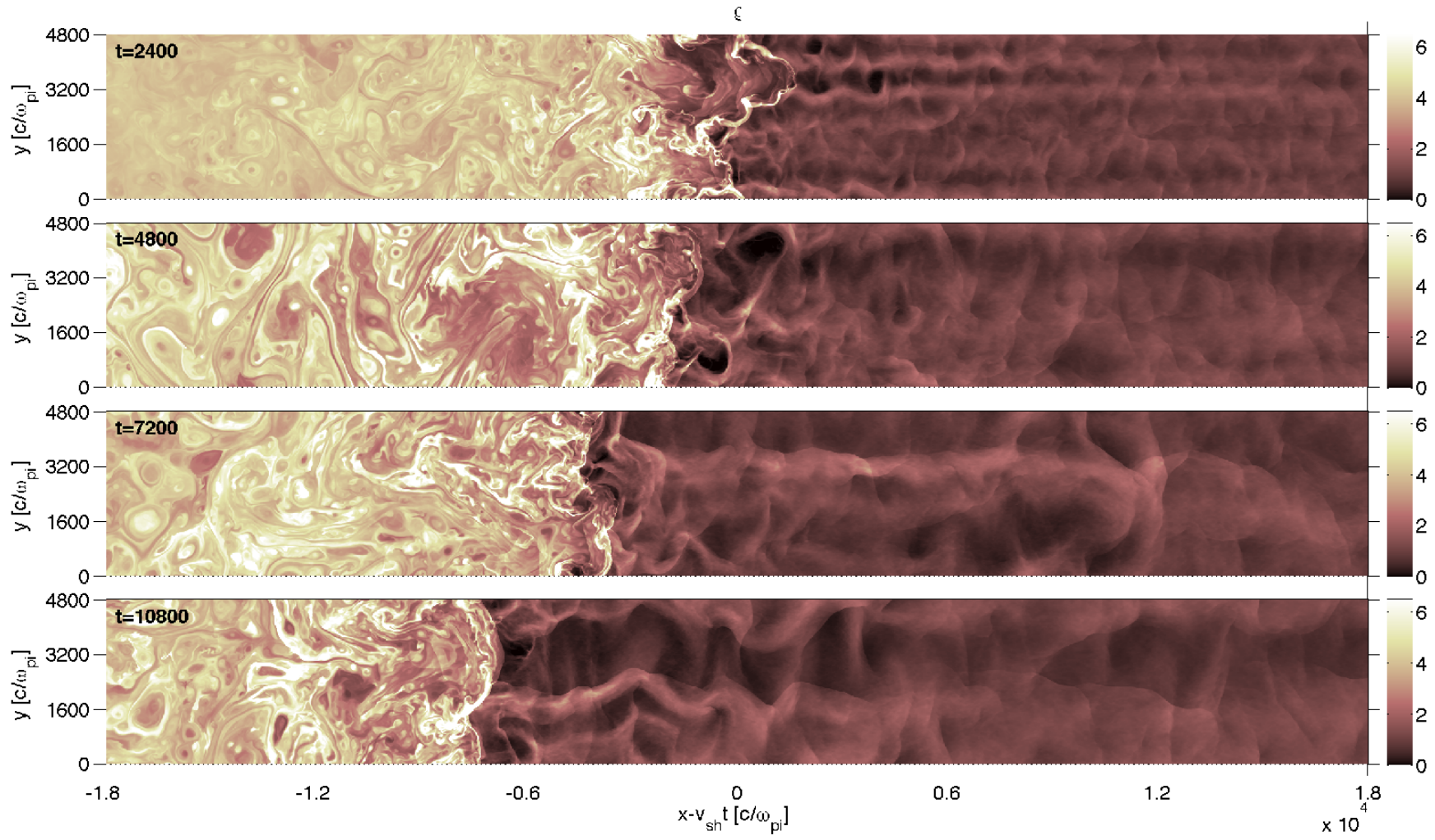

FIG. 9.- Snapshots of gas density at four different times (as indicated) from our run R2-REL with reduced speed of light $\mathbb{C}=10 \sqrt{2} v_{0}$ at four different times as labeled in each panel (unit is $\Omega_{c}^{-1}$ ). Only a small fraction of the simulation box is shown which is adjusted to cover the vicinity of the expected shock location at $x=v_{s h} t=v_{0} t / 3$.

In Figure 11, we show the evolution of weighted maximum particle energy. We first note that acceleration is slower than our fiducial non-relativistic run R2 (indicated in black dash-dotted line). This is most likely due to the reduction in scattering efficiency as particle transitions into relativistic regime (e.g., smaller gyro-frequency, and gyro-radius increases with energy more quickly), resulting in slower acceleration. Since the threshold energy of $E=200 E_{s h}$ is achieved at very early stage of the simulation with $t<1000 \Omega_{c}^{-1}$, the bulk of the growth in $E_{\max }$ occurs in the relativistic regime (note that our definition of $E_{\max }$ is a factor of several larger than the cutoff energy). In addition, we find that $E_{\max }$ increases with time faster at early times $\left(t \lesssim 3000 \Omega_{c}^{-1}\right)$, but becomes slower later. We therefore provide two linear fits for $t<3000 \Omega_{c}^{-1}$ and $t>4000 \Omega_{c}^{-1}$ respectively, where the slopes differ by a factor of $\sim 2$. The difference most likely related to the reduction of CR current as escaping particles gradually become dominated by relativistic particles, which in turn affects the strength of the turbulence. While more detailed analysis of particle diffusion is beyond the scope of this work, we have performed further experiments with larger and smaller particle speed of light, and confirm the effects discussed above.

\section{SUMMARY AND DISCUSSION}

In this paper, we have rigorously derived an MHD formulation which describes the interaction between collisionless CRs and a thermal plamsa. Backreaction from
CRs to the thermal plasma is incorporated in the form of momentum, energy and electromagnetic feedback, as Equations (19), (20) and (15). While momentum and energy feedback have been well understood as a result of momentum and energy conservation in the composite system of CRs and gas, we point out the previously neglected electromagnetic feedback, which we term as the CR-induced Hall (CR-Hall) term. It becomes important when the relative drift velocity between electrons and ions induced by $\mathrm{CR}$ streaming approaches the Alfvén velocity, or their ratio $\Lambda$ defined in (12) approaches order unity. Our formulation is applicable (i) on scales much larger than the ion inertial length $c / \omega_{p i}$, which is the scale that MHD is considered applicable to describe thermal plasma, and (ii) when CRs constitute a negligible fraction of the total gas mass (which is almost always the case in real systems). While our formulation remains valid when $\Lambda \gtrsim 1$ (i.e., extremely strong CR current), additional plasma effects may take place that are not captured in the formulation.

We have implemented this formulation in the Athena MHD code, where CRs are treated as kinetic particles using the PIC technique. An artificial speed of light $\mathbb{C}$ can be specified for $\mathrm{CR}$ particles so that they can be either non-relativistic or fully relativistic, as long as $\mathbb{C}$ is much larger than any MHD velocity. All ingredients of our MHD-PIC code are well tested with carefully designed problems and have achieved excellent performance. Note that for conventional full PIC or hybrid- 


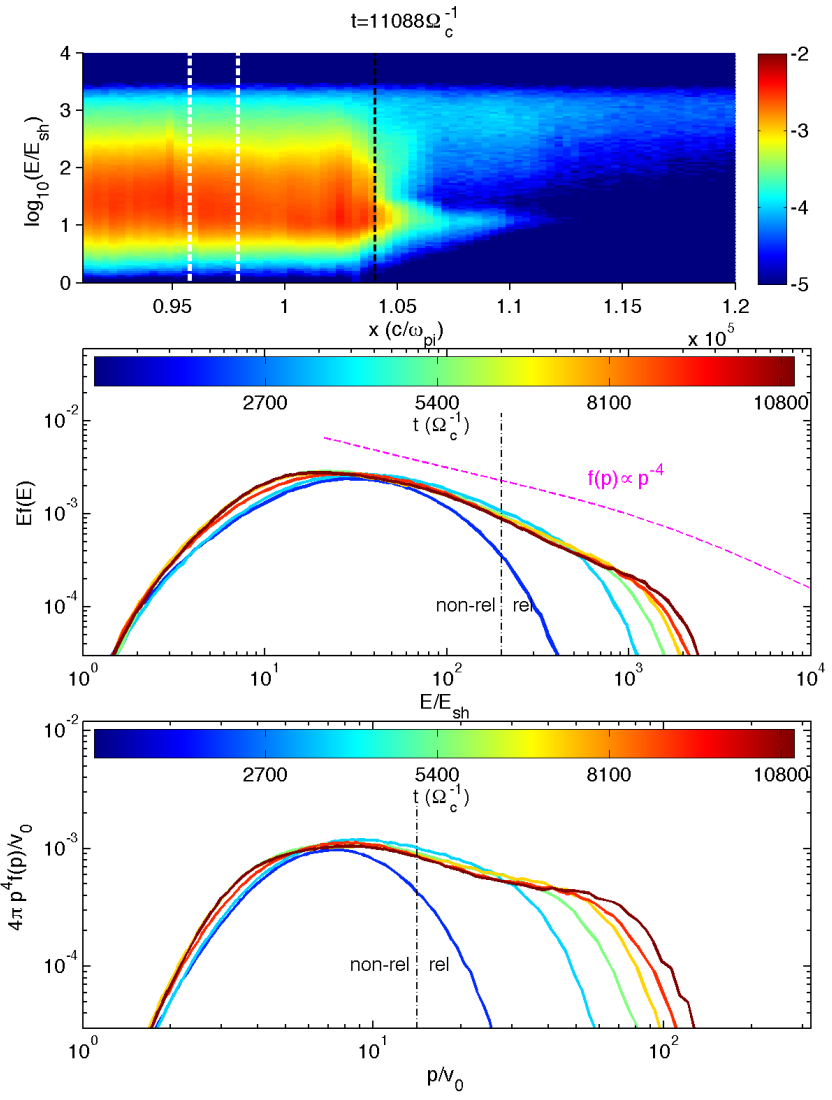

FIG. 10.- Energy and momentum spectrum of the injected CR particles from our run R2-REL with reduced speed of light $\mathbb{C}=10 \sqrt{2} v_{0}$. The top panel shows the energy spectrum as a function of $x$ at time $t=11088 \Omega_{c}^{-1}$ (about the end of the run) in units of $E_{s h}=v_{s h}^{2} / 2$. The middle (bottom) panel shows the time evolution of the downstream particle energy (momentum) spectrum as marked with different colors indicated by the color table. The energy and momentum spectra are shown in dimensionless form $E f(E)$ and $\left.4 \pi p^{4} f(p) / v_{0}\right)$, respectively. The spectrum is extracted by averaging through a layer at a fixed distance behind the shock front. As an example, vertical black dashed lines in the top panels mark the location of the shock front, and the vertical white dashed lines mark the layer where the downstream spectrum is extracted. The dash-dotted lines in the middle and bottom panels mark the transition from non-relativistic to relativistic regimes. In the middle panel, the expected energy spectrum from a $f(p) \propto p^{-4}$ momentum spectrum is also shown.

PIC codes, the requirements to accommodate the large Larmor radius and diffusion length of the $\mathrm{CR}$ particles and to resolve microphysical plasma scales (on the order of the ion or electron inertial length) become increasingly incompatible with higher CR energy. By circumventing the tiny plasma scales, our MHD-PIC approach enables us to study the physics of CR-gas interaction on much larger, potentially macroscopic, scales with dramatically reduced computational cost, while it still retains the full kinetic nature of the $\mathrm{CR}$ particles.

Our MHD-PIC code is well suited to investigate a number of astrophysical problems, as discussed in Section 1. In this paper, we have mainly focused on particle acceleration in non-relativistic shocks, where the Bell instability plays a dominant role in exciting upstream turbulence and scattering CR particles. We first point out that when the CR-Hall term is properly taken into account,

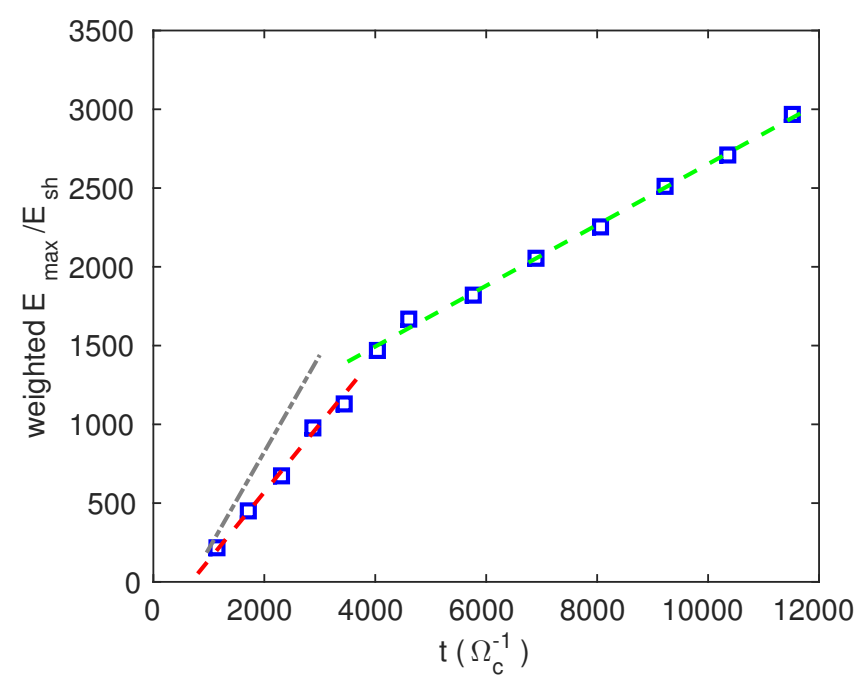

FIG. 11. - Time evolution of the weighted maximum particle energy $\bar{E}_{\text {max }}$ from our relativistic run R2-REL. Also shown in dashed lines are linear fits to data at $t<3000 \Omega_{c}^{-1}$ (red) and $t>4000 \Omega_{c}^{-1}$ (green). The black dash-dotted line is a reproduction of the fitting result to run R2 in Figure 6: acceleration is slower in the relativistic case.

the growth rate of the most unstable mode of the Bell instability is reduced with increasing $\Lambda$, and it is eventually overtaken by the filamentation instability upon $\Lambda \gtrsim 1$, which explains the findings by Riquelme \& Spitkovsky (2009). We also show that the transition occurs when the most unstable wavelength reaches the scale of ion inertial length, which is also at the limit of where our formulation is applicable.

As a proof-of-concept study and non-linear code test, we performed a set of simulations of parallel MHD shocks with Alfvén Mach number $M_{A}=30$, using an artificial particle injection prescription with fixed injection efficiency $\eta$. We observe the development of the Bell instability followed by filamentation and cavitation as the upstream flow approaches the shock front, leading to strong magnetic field amplifications and vorticity generation. We find efficient acceleration of the CR particles, which quickly develop a power-law tail in the energy spectrum with the expected slope of $f(E) \propto E^{-3 / 2}$ when all particles are non-relativistic. The maximum particle energy is found to increase linearly with time, consistent with efficient particle scattering in the Bohm limit. All these features are consistent with findings from recent hybrid simulations (Caprioli \& Spitkovsky 2014a,b,c), justifying the ability of our MHD-PIC approach at capturing the non-linear evolution of CR-driven instabilities and Fermi acceleration in non-relativistic shocks. We also show that the CR-Hall term can become important in the vicinity of the shock front in the upstream, especially in the cavities, and for high Mach number shocks. On the other hand, the CR-Hall effect becomes negligible in the shock far upstream.

By choosing a reduced particle speed of light $\mathbb{C}$, we further perform a shock simulation with substantially larger simulation box and longer duration, and follow particle acceleration from non-relativistic regime to relativistic regimes. We confirm the expected momentum spectrum 
of $f(p) \propto p^{-4}$ in both regimes, with a small drop in normalization in the relativistic part of the spectrum. We find that maximum particle energy increases at a slower rate in the relativistic regime (yet still linear in time), which is likely due to less efficient particle diffusion. In addition, escaping $\mathrm{CR}$ particles are less effective current carriers as they become relativistic, leading to weaker upstream turbulence and slower particle acceleration.

A major limitation of our initial study of particle acceleration in non-relativistic shocks is that particle injection is handled artificially. We find that with relatively small injection efficiency $\eta=10^{-3}$, the shock is less perturbed and particle acceleration efficiency is low $(\xi \sim 6 \%)$ by the end of the run. The shock become over-smoothed with large injection efficiency $\eta=4 \times 10^{-3}$ accompanied by rapid acceleration of particles to $\xi \gtrsim 20 \%$. Our fiducial choice of $\eta=2 \times 10^{-3}$ appears to be optimal and gives acceleration efficiency $\xi \sim 13-15 \%$, comparable with self-consistent hybrid-PIC simulations. In reality, we expect particle injection to be self-adapted to the level such that the shock is not over-smoothed, maintaining particle acceleration efficiency at some modest level. In addition, we also expect $\eta$ to gradually decrease with time as particles are accelerated to higher energies, because the energy content of the power-law spectrum would diverge with increasing maximum particle energy. Therefore, our conclusion of optimal injection efficiency $\eta \approx 2 \times 10^{-3}$ mainly applies to the initial stage of particle acceleration. Nonetheless, for this effect to be prominent, the CR energy must span several decades in the energy spectrum, which is not easily achieved in current simulations.

In the future, we will aim to re-design the particle injection prescriptions in a way that mimics the particle injection process in hybrid-PIC simulations. Our current simple prescription fully relies on the shock detection and tracking algorithm, and its details may have subtle long-term feedback to the shock structure in the vicinity of the shock front. Such dependence will be relieved in the new prescriptions. Once properly calibrated with hybrid-PIC simulations, we will be able to systematically explore the parameter space of particle acceleration in non-relativistic shocks, including Alfvénic Mach number and magnetic obliquity, and follow the long-term evolution of the shocks towards larger scales. In particular, the Alfvénic Mach number in supernova remnant shocks is typically several hundred or higher, which is out of reach of current hybrid-PIC simulations, but will become feasible with our MHD-PIC approach.

We thank the anonymous referee for a very useful report that led to several improvements to the paper, particularly on code implementation, convergence checks, and overall clarity. X.-N.B is supported by NASA through Hubble Fellowship grant HST-HF2-51301.001A awarded by the Space Telescope Science Institute, which is operated by the Association of Universities for Research in Astronomy, Inc., for NASA, under contract NAS 5-26555. D.C. is supported by NASA grant NNX14AQ34G. L.S. is supported by NASA through Einstein Fellowship grant number PF1-120090 awarded by the Chandra X-ray Center, which is operated by the Smithsonian Astrophysical Observatory for NASA under contract NAS8-03060. This work was partially supported by Simons Foundation (grants 267233 and 291817 to AS), and was facilitated by Max Planck/Princeton Center for Plasma Physics. Computation for this work was performed on computational resources supported by PICSciE and the OIT's High Performance Computing Center at Princeton University. This work also used resources on Stampede at Texas Advanced Computing Center through XSEDE grant TG-AST140001.

\section{APPENDIX}

\section{A: IMPLEMENTATION OF THE MHD-PIC SCHEME: DETAILS}

\section{"Mass" of CR particles}

In our implementation, each CR particle is assigned a "mass" $m_{\mathrm{CR}}$, which in reality represents the CR mass density carried by the particle (as a swarm of real particles). The charge and current density carried by particle $j$ in the code is connected to its "mass" again via the $q / m c$ factor

$$
\begin{aligned}
& n_{\mathrm{CR}, j} / c=(q / m c)_{j} m_{\mathrm{CR}, j}, \\
& \boldsymbol{J}_{\mathrm{CR}, j} / c=(q / m c)_{j} m_{\mathrm{CR}, j} \boldsymbol{u}_{j} .
\end{aligned}
$$

The total CR charge and current density $n_{\mathrm{CR}}$ and $\boldsymbol{J}_{\mathrm{CR}}$ at all grid points can be obtained by particles deposits using the TSC scheme.

We also define the charge-to-mass ratio $(q / m c)_{g}$ for the thermal ions in the gas, which is treated to be independent from that for the CR particles. The charge density of the thermal ions $n_{i}$ (or $n_{g}$ ) is then obtained from the gas density $\rho$ via

$$
n_{g} / c=(q / m c)_{g} \cdot \rho .
$$

The charge density ratio factor $R$ defined in (10) can then be readily obtained based on (A1) and (A2).

\section{The Particle Integrator}

We briefly describe our implementation of the Boris integrator and how it is coupled with the MHD integrator. Let $\boldsymbol{x}_{j}^{(n)}$ and $\boldsymbol{v}_{j}^{(n)}$ be the location and velocity of the $j$ th particle at step $n$. The integration scheme to step $n+1$ follows 
the leap-frog pattern in the form of "drift-kick-rotate-kick-drift":

$$
\begin{aligned}
\boldsymbol{x}_{j}^{(n+1 / 2)} & =\boldsymbol{x}_{j}^{(n)}+\frac{1}{2} \boldsymbol{u}_{j}^{(n)} \Delta t, \\
\boldsymbol{v}_{j}^{0} & =\boldsymbol{v}_{j}^{(n)}+\frac{h}{2} c \mathcal{E}_{j}^{\left(n+\frac{1}{2}\right)} \\
\boldsymbol{v}_{j}^{1} & =\boldsymbol{v}_{j}^{0}+2 \frac{\boldsymbol{v}_{j}^{0}+\boldsymbol{v}_{j}^{0} \times \boldsymbol{b}}{1+b^{2}} \times \boldsymbol{b}, \\
\boldsymbol{v}_{j}^{(n+1)} & =\boldsymbol{v}_{j}^{1}+\frac{h}{2} c \mathcal{E}_{j}^{\left(n+\frac{1}{2}\right)} \\
\boldsymbol{x}_{j}^{(n+1)} & =\boldsymbol{x}_{j}^{(n+1 / 2)}+\frac{1}{2} \boldsymbol{u}_{j}^{(n+1)} \Delta t
\end{aligned}
$$

where $h \equiv\left(q / m_{j} c\right) \Delta t, \boldsymbol{b} \equiv h \boldsymbol{B}_{j}^{(n+1 / 2)} / \gamma_{j}$, and $\gamma_{j}$ is the Lorentz factor for $\boldsymbol{v}_{j}^{0}$ and $\boldsymbol{v}_{j}^{1}$ (which are the same). Note the relation between $\boldsymbol{u}_{j}$ and $\boldsymbol{v}_{j}$ defined in (22).

Electromagnetic fields are extracted from the half time step in the MHD integrator (with electric field modified by the CR-Hall term), which we denote as $\mathcal{E}^{(n+1 / 2)}$ and $\boldsymbol{B}^{(n+1 / 2)}$. In addition, while cell-centered field satisfies $\mathcal{E} \cdot \boldsymbol{B}=0$, this is not guaranteed for the interpolated fields, which may cause spurious particle acceleration. Therefore, we always clean the parallel component of the interpolated electric field $\mathcal{E} \rightarrow \mathcal{E}-\mathcal{E} \cdot \boldsymbol{B} / B$ before it is applied to push the particles.

The use of mid-point electromagnetic field (which is fixed during the integration) and the symmetric integration scheme guarantees second-order accuracy in time. The Boris integrator is time-reversible, and it preserves the geometric properties of particle gyration in the absence of electric field. Although being straightforward, we do not implement the Vay's integrator (Vay 2008). While it better conserves particle energy in the ultra-relativistic regime and when the electric field strength approaches the magnetic field strength, it is computationally more expensive. Moreover, in non-relativistic $\mathrm{MHD}$, we always have $|c \mathcal{E}| \ll|\mathbb{C} B|$ (by construction via the choice of $\mathbb{C}$ ). In this regime, the Vay pusher behaves essentially the same as the Boris pusher.

\section{The MHD-PIC Integration Scheme}

We now describe the way to combine the particle integrator with the MHD integrator in Athena that achieves secondorder accuracy in the composite system. For our purpose, the second-order CTU MHD integrator in Athena can be considered as a simple predictor-corrector scheme. Our MHD-PIC scheme is built on top of the existing predictorcorrector framework. To simplify the notation, we use $f$ and $g$ to represent particle and gas quantities respectively, and their evolution satisfies the differential equations

$$
\frac{d f}{d t}=F(f, g), \quad \frac{d g}{d t}=G(f, g) .
$$

The former describes particle equation of motion under the electromagnetic field provided by the gas, and the latter represents MHD equations with CR feedback. Using this notation, our integration scheme can be expressed as follows

$$
\begin{aligned}
g^{(n+1 / 2)} & =g^{(n)}+G\left(f^{(n)}, g^{(n)}\right) \frac{\Delta t}{2}, \\
f^{(n+1)} & =f^{(n)}+F\left(f^{(n+1 / 2)}, g^{(n+1 / 2)}\right) \Delta t, \\
g^{(n+1)} & =g^{(n)}+G\left(\frac{f^{(n)}+f^{(n+1)}}{2}, g^{(n+1 / 2)}\right) \Delta t .
\end{aligned}
$$

In the above, $(\mathrm{A} 5 \mathrm{~b})$ represents the particle integrator described in the previous subsection. Below, we mainly focus on (A5a) and (A5c), which represent the predictor and corrector steps for the gas evolution with CR feedback.

The basic procedure to treat backreaction from CRs to the gas involves adding source terms to the gas equations by depositing relevant physical quantities (momentum, energy, etc.) from the location of individual particles to neighboring grid cell centers. In the predictor step (A5a), the CR feedback is computed using the first equality of Equations (19) and (20): we deposit the charge and current densities of individual particles from their initial positions $\boldsymbol{x}_{j}^{(n)}$ to the grid to obtain $n_{\mathrm{CR}}^{(n)}$ and $J_{\mathrm{CR}}^{(n)}$, as well as $R^{(n)}$ based on the gas density $\rho^{(n)}$. Together with MHD quantities $\mathcal{E}_{0}^{(n)}$ and $\boldsymbol{B}^{(n)}$, the momentum and energy feedback are directly calculated at cell centers.

In the corrector step, we take advantage of the fact that all particles have evolved for a full time step, and compute the momentum and energy difference for each particle $j$ over one time step:

$$
\begin{aligned}
d \boldsymbol{M}_{j} & =m_{\mathrm{CR}, j}\left(\boldsymbol{v}_{j}^{(n+1)}-\boldsymbol{v}_{j}^{(n)}\right) . \\
d E_{k, j} & =m_{\mathrm{CR}, j}\left(E_{k, j}^{(n+1)}-E_{k, j}^{(n)}\right) .
\end{aligned}
$$


The opposite of these quantities are deposited to the gas, which account for momentum and energy feedback from CRs. The deposit is exerted from particle location at half step $\boldsymbol{x}_{j}^{(n+1 / 2)}$. This guarantees the consistency of our algorithm since the feedback is exerted at the same location as where the particle experiences the Lorentz force. With Athena being a Godunov code, exact conservation of total momentum and total energy of the composite gas-CR system is achieved. Moreover, this procedure also ensures our MHD-PIC algorithm to be second-order accurate.

Regarding the electromagnetic feedback due to the CR-Hall term, Equation (15) indicates that the correction to the electric field is directly proportional to the momentum feedback. Therefore, no additional particle deposits are needed and we simply correct the electric field and the energy flux due to the CR-Hall term based on the momentum feedback in both the predictor and corrector steps. The corrections are implemented in accordance with the intrinsic algorithm that the CTU integrator handles the induction and energy equations.

\section{Timestepping and Code Performance}

The timestepping for the MHD part of the code obeys the standard Courant-Friedrichs-Lewy condition. Additional constraints on the timestepping from particles include the following.

First, particle Larmor time $1 / \Omega_{L}=\gamma(q B / m c)^{-1}$ must be resolved. In practice, we define a number $\Upsilon$ so that we demand $\Omega_{L, i} \Delta t \leq \Upsilon$ for all particles. Our tests in Section B.1 suggest that $\Upsilon \lesssim 0.5$ is generally sufficient. For all shock simulations in this paper, we use $\Upsilon=0.3$, and we have also performed test simulations with $\Upsilon=0.1$ and 0.5 and do not find any practical difference in the outcomes.

Second, individual particles can not travel more than two grid cells per time step. This follows from the specifics of our MHD-PIC scheme ${ }^{12}$.

Since CR particles can become relativistic, with velocities much larger than typical fluid velocity, the code can be slowed down substantially. To reconcile this issue, we have implemented sub-cycling for the CR particles: we evolve multiple steps of particles per MHD step. In other words, the single-step particle evolution illustrated in (A5b) is now replaced by multiple steps of particle updates. When evolving the particles, we always use the electromagnetic field at half MHD step (i.e., $g^{(n+1 / 2)}$ ), hence the MHD-PIC scheme remains second-order accurate over a full MHD step. Particles deposit feedback at each sub-step, and the feedback accumulated from all sub-steps will finally be added to the gas in the corrector step as usual.

We have tested the code performance on a single CPU as well as in parallel. For particles, most of the computation time is spent on the Boris integrator, particle feedback and various interpolations. The serial test takes place on a Intel Xeon E5 $2.3 \mathrm{GHz}$ processor. Without sub-cycling, it takes about $1 \mu$ s to integrate one particle in $3 \mathrm{D}$, and $0.6 \mu$ s to integrate one particle in 2D. Combined with isothermal MHD using the CTU integrator with third-order reconstruction and the HLLD solver, we find that the code spends roughly equal amount of time on the MHD integrator and on the particles when using approximately 7 (for 3D) or 6 (for 2D) particles per cell. When run in parallel, because of extra steps for feedback exchange, the particle numbers quoted above are reduced to 6 (for 3D) and 5 (for 2D). The code performance on the particles can be enhanced by using sub-cycling. With the same test on the single processor, we find that the averaged time to integrate one particle for one particle sub-step is reduced by $30 \%$ when using 10 particle sub-steps per MHD step.

\section{B: CODE TESTS}

The two main ingredients of our MHD-PIC code are the Boris integrator and the particle feedback. We outline here two problems that test the two ingredients separately, and show that the code performs well in these respects. Since in Athena the units for magnetic field are such that magnetic permeability $\mu=1$, factors of $4 \pi$ will be left out here as well.

\section{Gyration Test}

We test the Boris integrator with the particle gyration problem. We set up a uniform background gas that moves at constant velocity $v_{0}$ in the $\hat{y}$ direction. The velocity can be "relativistic" based on the artificial speed of light $\mathbb{C}$, with Lorentz factor $\gamma_{0}=\mathbb{C} / \sqrt{\mathbb{C}^{2}-v_{0}^{2}}$. To facilitate analytical calculation, we prescribe other parameters in a frame that is co-moving with the gas. In the co-moving frame, the electric field is zero, and there is a uniform magnetic field $B_{0}$ along the $\hat{x}$ direction. We include one particle whose parallel and perpendicular four-velocities in the comoving frame are given by $v_{\|}$and $v_{\perp}$, with Lorentz factor $\gamma_{1}=\sqrt{\mathbb{C}^{2}+v_{\|}^{2}+v_{\perp}^{2}} / \mathbb{C}$. Then we have the Larmor radius $R_{L}=v_{\perp}\left(q B_{0} / m c\right)^{-1}$, gyro-frequency $\Omega_{L}=\left(q B_{0} / \gamma_{1} m c\right)$, parallel velocity $u_{0}=v_{\|} / \gamma_{1}$. We compute the particle orbit in the lab frame using the code and compare the results with analytical orbits in the co-moving frame.

In Figure 12, we show the test results for non-relativistic particles. We initialize the problem with $B_{0}=q / m c=$ $v_{\perp}=1, v_{\|}=0, \mathbb{C}=10$, and hence $R_{L}=1$ and $\Omega_{L}=1$. Considering the fact that the time step in our MHD-PIC code is generally not a constant, the time step we adopt for this test is $\Delta t=0.5+0.1 \cos \alpha$, where $\alpha$ is a random number. Two tests are performed, one with zero drift velocity $v_{0}=0$ (blue dashed), and the other with drift velocity $v_{0}=1$ (red

\footnotetext{
12 With the particle module turned on in Athena, the innermost two (otherwise just one) ghost zones are updated during the pre-
}

dictor step. The TSC scheme requires that particles can move to the innermost but not the next ghost zone in the predictor step. 

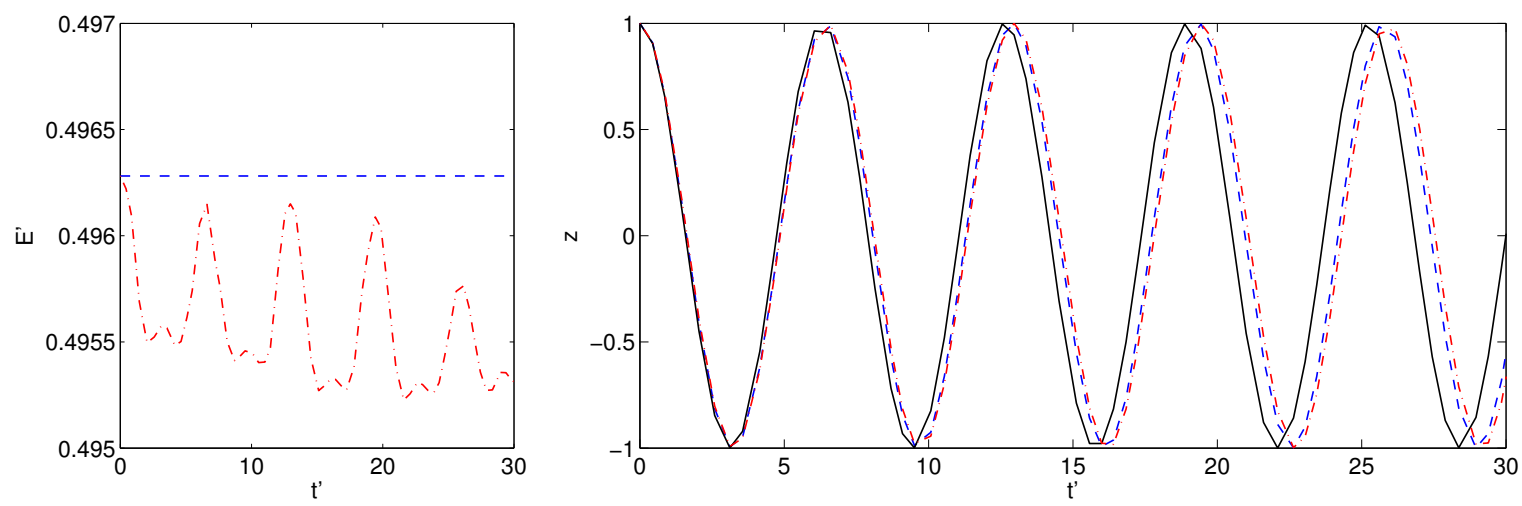

FIG. 12. - Gyration test for non-relativistic particle orbits with $v_{\perp}=0.1 \mathbb{C}=1$ using variable time step with $\Omega_{L} \Delta t=0.5 \pm 0.1$. Left: time evolution of particle kinetic energy in the co-moving frame (indicated by the prime). Right: evolution of particle position in the co-moving frame. Blue dashed lines correspond to zero background flow velocity $\left(v_{0}=0\right)$, red dash-dotted lines correspond to mildly relativistic drift of the background gas $\left(v_{0}=1\right)$. Black solid lines represent analytical results. Note that we have chosen to a large time step to exaggerate the truncation errors.
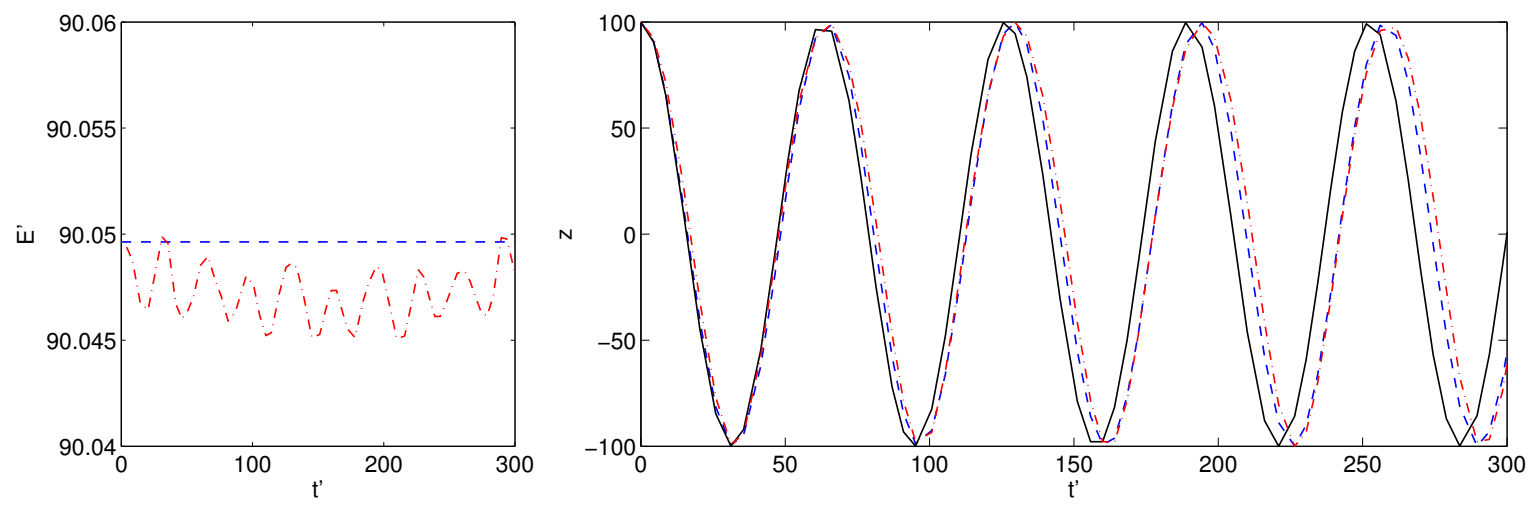

FIG. 13. - Same as Figure 12, but for relativistic particles with $v+=10 \mathbb{C}$.

dash-dotted). Shown on the left is the evolution of particle kinetic energy in the co-moving frame, while on the right is the particle trajectory in the co-moving frame. We see that for $v_{0}=0$, the integrator conserves energy exactly despite the variable time step, with relatively small phase error. With drift $v_{0}=1$, energy is no longer conserved. There is systematic oscillation in $E^{\prime}$ is at $0.1 \%$ level, which is due to non-zero background electric field. ${ }^{13}$ The energy $E^{\prime}$ also undergoes a random-walk type drift over time, which is due to the variable time step. Note that both oscillation and drift in $E$ are inherent to the Boris pusher under these circumstances.

In Figure 13, we show the results from the same test but for relativistic particles. We increase particle velocity to $v_{\perp}=100\left(\gamma_{1} \approx 10\right)$, keeping other parameters fixed. Correspondingly, we have $R_{L}=100$ and $\Omega_{L} \approx 0.1$, and we modify the time step to $\Delta t=5 \pm \cos \alpha$ so that $\Omega_{L} \Delta t$ remains the same as before. We see again that without $E \times B$ drift, the integrator captures particle orbits perfectly well. In the presence of drift, the fractional error in the energy is smaller than in the previous case, while the phase error remains similar.

Note that we have used relatively large time step $\left(\Omega_{L} \Delta t=0.5\right)$ to exaggerate the truncation errors. The time step in real applications is typically much smaller. Moreover, random variation in the timestep also enhances energy drift in the tests, while in real simulations, timestep variation is smooth. Therefore, our particle gyration tests have demonstrated a reliable performance of the Boris integrator for astrophysical applications.

\section{Linear Growth of the Bell Instability}

We test the CR feedback and the CR-Hall term using the linear dispersion relation of the Bell instability, as discussed in Section 4. The test is conducted by evolving the exact eigen-vectors of the most unstable mode.

Two sets of tests are performed. In the first test, we suppress the CR-Hall term by using sufficiently small $R$ such that $\Lambda \equiv R U_{s} / v_{A} \ll 1$. In this case, the most unstable wavenumber is $k_{0}$, and the dispersion relation (29) gives

$$
\omega=k_{0} v_{A}\left(\epsilon+\mathrm{i} \sqrt{1-\epsilon^{2}}\right)=k_{0} v_{A} \exp \left[\mathrm{i}\left(\frac{\pi}{2}-\theta\right)\right]
$$

13 The level of energy oscillation increases rapidly with increas-
gas flow velocity $v_{0}$ once $v_{0}$ exceeds particle velocity. Never- theless, for all potential applications of our code, we expect $\mathrm{CR}$ particle velocity to be (much) larger than $v_{0}$. 
$1 \mathrm{D}$

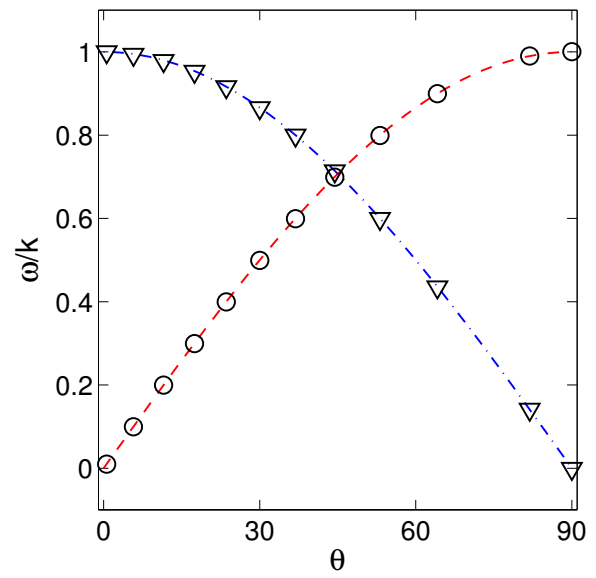

2D

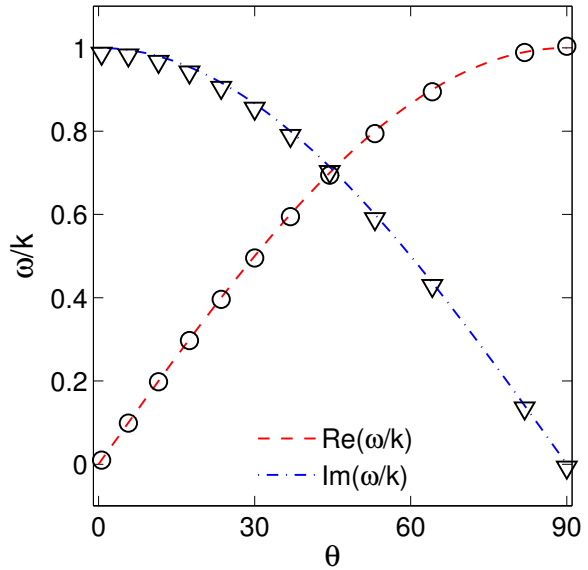

3D

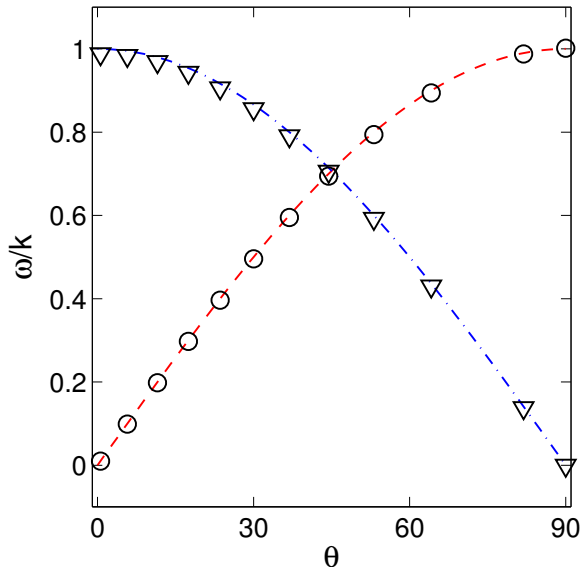

FIG. 14.- Linear dispersion relation test of the Bell instability in 1D, 2D and 3D with $\Lambda \ll 1$ (the CR-Hall term is negligible). The real (red dashed and circles) and imaginary (blue dashh-dotted and triangles) parts of the most unstable mode as a function of $\theta$, defined as $\sin \theta=\epsilon$, where $\epsilon \equiv v_{A} / u_{0}$, the ratio of Alfvén velocity to the CR drift velocity. Symbols denote measured values from the code test, curves represent analytical expectations. See Section B.2 for details.
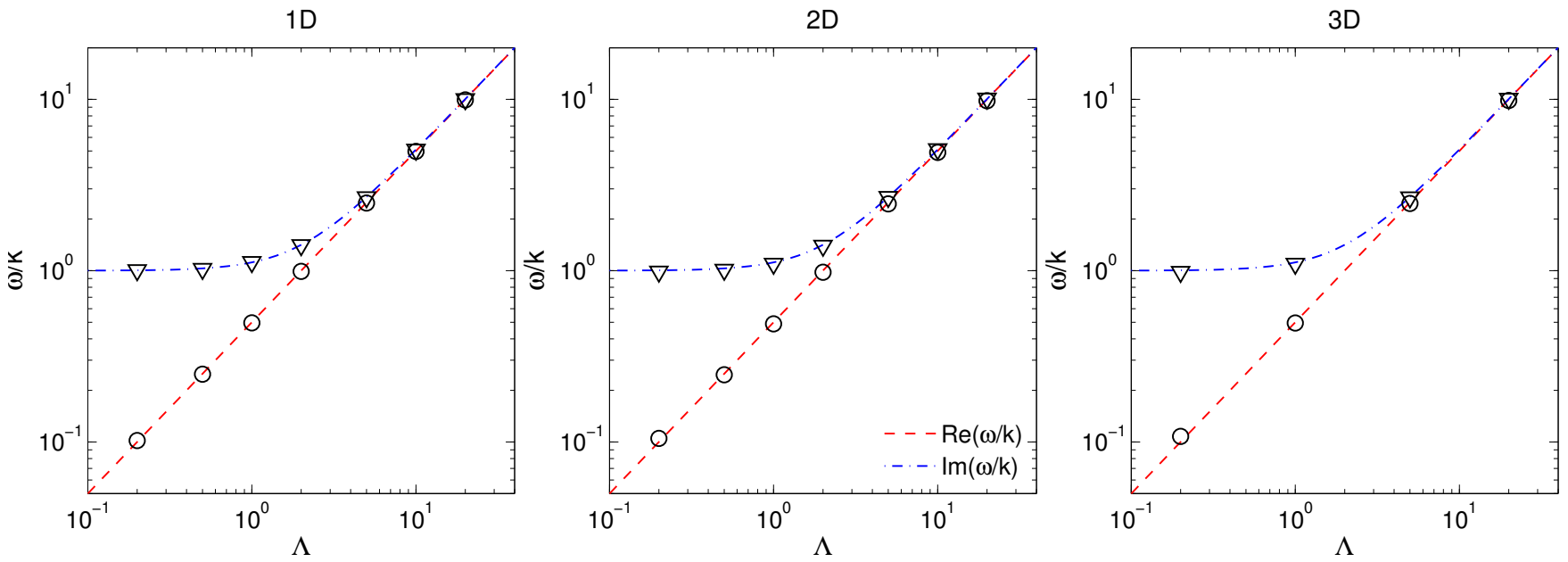

FIG. 15. - Linear dispersion relation test of the Bell instability in $1 \mathrm{D}, 2 \mathrm{D}$ and $3 \mathrm{D}$ with $\epsilon \ll 1$ (the CR charge density is negligible). The real (red dashed and circles) and imaginary (blue dashh-dotted and triangles) parts of the most unstable mode as a function of $\Lambda$, defined as $\Lambda=R / \epsilon$. Symbols denote measured values from the code test, curves represent analytical expectations. See Section B.2 for details.

where we have defined $\sin \theta \equiv \epsilon$. The corresponding eigenvector of this mode reads

$$
\begin{aligned}
& b_{y}=b_{\perp} \cos \left(k_{0} x-\epsilon k_{0} v_{A} t\right), \\
& b_{z}=b_{\perp} \sin \left(k_{0} x-\epsilon k_{0} v_{A} t\right), \\
& u_{y}=\frac{v_{A}}{B_{0}} b_{\perp} \sin \left(k_{0} x-\theta-\epsilon k_{0} v_{A} t\right), \\
& u_{z}=-\frac{v_{A}}{B_{0}} b_{\perp} \cos \left(k_{0} x-\theta-\epsilon k_{0} v_{A} t\right),
\end{aligned}
$$

where $\boldsymbol{b}$ and $\boldsymbol{u}$ denote transverse perturbations of the magnetic field and fluid velocity, and the wave amplitude grows as $b_{\perp} \propto \exp \left(\cos \theta k_{0} v_{A} t\right)$.

To setup the problem, we consider background gas with uniform density $\rho_{0}$ and zero velocity. Let $\boldsymbol{B}_{0}$ be the background magnetic field, $\boldsymbol{J}_{\mathrm{CR}}=n_{\mathrm{CR}} \boldsymbol{u}_{0}$ be the external uniform current density provided by the CR particles, both of which are along the $\hat{x}$ direction. Note that the CR current is provided by a spatially uniform distribution of CRs with number density $n_{\mathrm{CR}}$ that drift at velocity $u_{0}$. For our code test purpose, we treat the CR particles as having infinite inertia so that the their effects are simply to provide constant external current and charge.

In our code test, we set up the exact eigenvector of the most unstable mode (B2), and test the dispersion relation 
(B1) as a function of $\epsilon$ (or $\theta)$. To elaborate, we consider the following parameters: $B_{0}=\rho_{0}=1$ (hence $v_{A}=1$ ). We fit one most unstable wavelength $\lambda_{0}$ into the simulation box, with $\lambda_{0}$ determined by the box size set by the user. This fixes the CR current density: $J_{\mathrm{CR}}=2 B_{0} k_{0}=4 \pi / \lambda_{0}$. We initiate with a cold population of particles moving at constant velocity $u_{0}=v_{A} / \epsilon$ along the direction of $\boldsymbol{B}_{0}$. There is one particle per grid cell located cell centers. The main parameter is $\epsilon$ ranging from 0 to 1 . Correspondingly, the growth rate of the instability is simply $\sqrt{1-\epsilon^{2}} k_{0}$. In order for the Lorentz force to be negligible on particle motion over a few $e$-folding time, we set $q / m c \equiv 10^{-6} k_{0}$ for the $\mathrm{CR}$ particles. This further determines the value of $m_{\mathrm{CR}}$, which is simply given by $m_{\mathrm{CR}}=J_{\mathrm{CR}} /\left[(q / m c) u_{0}\right]=2 \times 10^{6} \epsilon$. We finally set the gas isothermal sound speed to be $c_{s}=1$, and speed of light $\mathbb{C}=100 u_{0}$, both of which are irrelevant for this problem but needed in the code.

The problem generator deals with all 1D, 2D and 3D cases, where in multi-dimensions, the wave vector of the mode is not grid-aligned. The box size in these cases are $1, \sqrt{5} \times \sqrt{5} / 2,3 \times 1.5 \times 1.5$, resolved by $32,64 \times 32$ and $96 \times 48 \times 48$ cells respectively. The choice of such box size and resolution guarantees that $\lambda_{0}=1$ with approximately 32 cells per wavelength. We vary $\epsilon$ from 0.01 to 1.0 , and show the measured real and imaginary parts of $\omega / k$ in Figure 14 as a function of $\theta$ where $\sin \theta=\epsilon$. Theoretically, the real and imaginary parts should be exact sine and cosine curves, and we see that in all three dimensions the code very well reproduces the analytical phase velocity and growth rate. Although not obvious in the plot, deviations from analytical results in 2D and 3D cases are comparable and larger than the $1 \mathrm{D}$ case, but are well within $2 \%$.

In the second test, we relax the constraint on $\Lambda$ while keeping $R \ll 1$, which enables the CR-Hall term without violating our formulation. We also keep $\epsilon \ll 1$. In this case, the most unstable wavenumber is $k_{m}=k_{0} / f$, where $f=1+(\Lambda / 2)^{2}$, and the dispersion relation reads

$$
\omega=\left(k_{m} v_{A}\right)(\Lambda / 2+\mathrm{i} \sqrt{f}) .
$$

The corresponding eigen-vectors are

$$
\begin{aligned}
& b_{y}=b_{\perp} \cos \phi, \\
& b_{z}=b_{\perp} \sin \phi, \\
& u_{y}=\frac{v_{A}}{B_{0}} b_{\perp}[(\Lambda / 2) \cos \phi+\sqrt{f} \sin \phi], \\
& u_{z}=\frac{v_{A}}{B_{0}} b_{\perp}[(\Lambda / 2) \sin \phi-\sqrt{f} \cos \phi],
\end{aligned}
$$

where $\phi \equiv k_{m}\left[x-(\Lambda / 2) v_{A} t\right]$, and the wave amplitude grows as $b_{\perp} \propto \exp \left(k_{m} v_{A} \sqrt{f} t\right)$.

We use the same numerical setup as the previous test, except for resetting the eigenvectors. By varying $\Lambda$ from 0.2 to 20, we show the measured real and imaginary parts of $\omega / k$ in Figure 15 . We see that for all values of $\Lambda$, the phase velocity and growth rate are accurately reproduced from the regime $\Lambda \ll 1$ to $\Lambda \gg 1$. In particular, the measured growth rates deviate from analytical results by less than $0.5 \%$ for all cases at our numerical resolution. Although our code is may not be physically reliable if $\Lambda \gtrsim 1$ (see Section 2.1), this test demonstrates the successful implementation of the CR-Hall term.

\section{C: LINEAR DISPERSION RELATION OF THE BELL INSTABILITY}

In this Appendix, we provide more detailed derivation of the linear dispersion relation of the Bell instability taking into account of the CR-Hall term, following Equation (28) in Section 4. We first define

$$
\tilde{\omega} \equiv \omega-(\Lambda / 2) v_{A} k, \quad \tilde{U}_{s} \equiv U_{s}(1-R / 2)
$$

and two factors

$$
g \equiv(1-R)(1-R / 2), \quad f \equiv 1-R+(\Lambda / 2)^{2}
$$

Now Equation (28) can be rewritten with the new variables

$$
\mathrm{i}\left(\tilde{\omega}^{2}-f k^{2} v_{A}^{2}\right)(\boldsymbol{b} \times \hat{x})+g \frac{k J_{\mathrm{CR}} v_{A}^{2}}{B_{0}}\left(1-\frac{\tilde{\omega}}{k \tilde{U}_{s}}\right) \boldsymbol{b}=0 .
$$

Non-trivial solution requires $b_{y}= \pm \mathrm{i} b_{z}$, corresponding to left/right circularly polarized modes, and it is straightforward to obtain the dispersion relation

$$
\tilde{\omega}^{2}-f k^{2} v_{A}^{2}= \pm g \frac{k J_{\mathrm{CR}} v_{A}^{2}}{B_{0}}\left(1-\frac{\tilde{\omega}}{k \tilde{U}_{s}}\right)
$$

To make it more instructive, we further define

$$
k_{0} \equiv \frac{J_{\mathrm{CR}}}{2 B_{0}}, \quad \epsilon \equiv \frac{v_{A}}{\tilde{U}_{s}},
$$


and the above dispersion relation can be rewritten as

$$
\left(\tilde{\omega} \pm \epsilon k_{0} v_{A}\right)^{2}=f\left(k \pm \frac{g}{f} k_{0}\right)^{2} v_{A}^{2}-\left(k_{0} v_{A}\right)^{2}\left(\frac{g^{2}}{f}-\epsilon^{2}\right),
$$

where the two \pm signs must be taken to be the same. It reduces to Equation $(29)$ when $R \ll 1$, or $g=1$.

\section{REFERENCES}

Ackermann, M., Ajello, M., Allafort, A., et al. 2013, Science, 339, 807

Amano, T., \& Hoshino, M. 2007, ApJ, 661, 190

Amato, E., \& Blasi, P. 2005, MNRAS, 364, L76

Amato, E., \& Blasi, P. 2009, MNRAS, 392, 1591

Bai, X.-N. \& Stone, J. M. 2010a, ApJS, 190, 297

-. 2010b, ApJ, 722, 1437

Bell, A. R. 1978, MNRAS, 182, 147

—. 2004, MNRAS, 353, 550

-. 2005, MNRAS, 358, 181

Bell, A. R., Schure, K. M., Reville, B., \& Giacinti, G. 2013, MNRAS, 431, 415

Beresnyak, A., Yan, H., \& Lazarian, A. 2011, ApJ, 728, 60

Berezhko, E. G., Volk, H. J. 1997, Astroparticle Physics, 7, 183

Birdsall, C. K. \& Langdon, A. B. 2005, Plasma Physics Via Computer Simulation (Taylor \& Francis Group, 2005)

Blandford, R. D. \& Ostriker, J. P. 1978, ApJ, 221, L29

Boris, J. P. 1970, in Proceedings of the Fourth Conference on Numerical Simulation Plasmas (Navel Research Laboratory, Washington, D.C.), pp. 3-67

Bowers, K. J. \& Albright, B. J. \& Yin, L \& Bergen B. \& Kwan, T. J. T. 2008, Physics of Plasmas, 15, 5

Caprioli, D. 2012, JCAP, 7, 38

Caprioli, D. \& Spitkovsky, A. 2013, ApJ, 765, L20

-. 2014a, ApJ, 783, 91

—. 2014b, ApJ, 794, 46

-. 2014c, ApJ, 794, 47

Caprioli, D., Pop, A. R., \& Spitkovsky, A. 2015, ApJ, 798, L28

Ellison, D. C., Moebius, E., \& Paschmann, G. 1990, ApJ, 352, 376

Ellison, D. C., Baring, M. G., \& Jones, F. C. 1995, ApJ, 453, 873

Fermi, E. 1949, Physical Review, 75, 1169

Fonseca, R. A., et al. 2002, Computational ScienceICCS 2002, 342

Gardiner, T. A. \& Stone, J. M. 2005, Journal of Computational Physics, 205, 509

-. 2008, Journal of Computational Physics, 227, 4123

Gargaté, L., Bingham, R., Fonseca, R. A., \& Silva, L. O. 2007, Computer Physics Communications, 176, 419

Gargaté, L. \& Spitkovsky, A. 2012, ApJ, 744, 67

Giacalone, J. 2004, ApJ, 609, 452

Giacalone, J., Burgess, D., Schwartz, S. J., \& Ellison, D. C. 1992 Geophys. Res. Lett., 19, 433

Guo, F., \& Giacalone, J. 2013, ApJ, 773, 158

Guo, X., Sironi, L., \& Narayan, R. 2014, arXiv:1406.5190
Johansen, A., Youdin, A., \& Mac Low, M. 2009, ApJ, 704,

Kang, H., Jones, T. W., \& Gieseler, U. D. J. 2002, ApJ, 579, 337

Kulsrud, R., \& Pearce, W. P. 1969, ApJ, 156, 445

Kunz, M. W., Stone, J. M., \& Bai, X.-N. 2014a, Journal of Computational Physics, 259, 154

Lazarian, A., \& Beresnyak, A. 2006, MNRAS, 373, 1195

Lee, S.-H., Ellison, D. C., \& Nagataki, S. 2012, ApJ, 750, 156

Lehe, R., Parrish, I. J., \& Quataert, E. 2009, ApJ, 707, 404

Lipatov, A. S. 2002, The hybrid multiscale simulation technology: an introduction with application to astrophysical and laboratory plasmas/ Alexander S. Lipatov. Berlin; New York: Springer, 2002. Scientific computation, ISSN 1434-8322

Lucek, S. G., \& Bell, A. R. 2000, MNRAS, 314, 65

Malkov, M. A. 1997, ApJ, 485, 638

Morlino, G., \& Caprioli, D. 2012, A\&A, 538, A81

Narayan, R., Özel, F., \& Sironi, L. 2012, ApJ, 757, L20

Niemiec, J., \& Ostrowski, M. 2004, ApJ, 610, 851

Niemiec, J., Pohl, M., Stroman, T., \& Nishikawa, K.-I. 2008, ApJ, 684,1174

Parker, E. N. 1992, ApJ, 401, 137

Quest, K. B. 1988, J. Geophys. Res., 93, 9649

Reville, B. \& Bell, A. R. 2012, MNRAS, 419, 2433

Reville, B., \& Bell, A. R. 2013, MNRAS, 430, 2873

Riquelme, M. A., \& Spitkovsky, A. 2011, ApJ, 733, 63

Riquelme, M. A. \& Spitkovsky, A. 2009, ApJ, 694, 626

Schure, K. M. \& Bell, A. R. 2013, ArXiv:1307.6575

Skilling, J. 1975, MNRAS, 172, 557

Spitkovsky, A. 2005, Astrophysical Sources of High Energy

Particles and Radiation, 801, 345

Stone, J. M. \& Gardiner, T. 2009, New Astronomy, 14, 139

Stone, J. M., Gardiner, T. A., Teuben, P., Hawley, J. F., \& Simon, J. B. 2008, ApJS, 178, 137

Strong, A. W., Moskalenko, I. V., \& Ptuskin, V. S. 2007, Annual Review of Nuclear and Particle Science, 57, 285

Vay, J.-L. 2008, Physics of Plasmas, 15, 056701

Vladimirov, A., Ellison, D. C., \& Bykov, A. 2006, ApJ, 652, 1246

Winske, D., Yin, L., Omidi, N., \& et al. 2003, Space Plasma Simulation, 615, 136

Youdin, A. N. \& Goodman, J. 2005, ApJ, 620, 459

Zachary, A. L., \& Cohen, B. I. 1986, Journal of Computational Physics, 66, 469

Zachary, A. L. 1987, Ph.D. Thesis

Zirakashvili, V. N., \& Aharonian, F. A. 2010, ApJ, 708, 965 